\title{
Totale Institutionen. Die Auseinandersetzung von Georges Aperghis mit Paul Klee, Robert Walser und Adolf Wölfli
}

\author{
Eine Vernetzung
}

In den Jahren 2001 bis 2007 beschäftigte sich der griechisch-französische Komponist Georges Aperghis mit drei Berner Künstlern, die sich zu Beginn der 193oer-Jahre in Bern beinahe begegnet wären: Paul Klee, Robert Walser und Adolf Wölfli. Er stellt sie in seinen Werken in neue Kontexte und thematisiert die totalen Institutionen, mit denen sie konfrontiert waren. Dieses Kapitel greift über Robert Walser hinaus, stellt den im deutschen Raum noch wenig rezipierten Komponisten Georges Aperghis ${ }^{1}$ vor und betrachtet das Schaffen von Klee, Walser und Wölfli im Kontext von Erving Goffmans Theorie totaler Institutionen.

\subsection{Einleitung}

Am 24. Januar 1929 tritt Robert Walser nach einer psychischen Krise in die Heil- und Pflegeanstalt Waldau bei Bern ein. Anderthalb Jahre später stirbt am 6. November 1930 in ebendieser Klinik der »Schreiber, Dichter, Zeichner, Componist « ${ }^{2}$ und Maler Adolf Wölfli, der dort 35 Jahre interniert war. ${ }^{3}$ Am 19. Juni 1933 wird Walser gegen seinen Willen in die Heil- und Pflegeanstalt Herisau im Kanton Appenzell-Ausserrhoden überführt; gleichzeitig wird ein Bevormundungsverfahren eingeleitet. Am 21. April desselben Jahres wird Paul Klee an der Kunstakademie Düsseldorf »mit sofortiger Wirkung « von seiner Professur beurlaubt. »Meine Herren, es riecht in Europa bedenklich nach Leichen «, hatte er seiner Düsseldorfer Arbeitsgruppe zum Abschied gesagt. ${ }^{4}$ Am Ende des Jahres, am Heiligabend 1933 kehrt Klee in sein Elternhaus in Bern

1 Spätestens mit der Vergabe des mit 250'ooo Euro dotierten Ernst von Siemens Musikpreis 2021 an Georges Aperghis dürfte sich das ändern.

2 Wölfli: Schreiber, Dichter, Zeichner, Componist.

3 Es gibt keinen Hinweis darauf, dass sich Adolf Wölfli und Robert Walser in der Anstalt begegnet sind. Vgl. Wendler: Wölfli und Walser, S. 208.

4 Michels: Paul Klee, S. 96. 
zurück. In seinem Gepäck ist auch ein Teil der Handpuppen, die er zwischen 1916 und 1923 für seinen Sohn Felix gebaut hatte. Kurz nach seiner Ankunft bemüht sich Paul Klee um den Erhalt der schweizerischen Staatsbürgerschaft. Das Verfahren zieht sich hin, und Klee erliegt einige Tage vor der definitiven Zuerkennung seiner schweren Krankheit.

$\mathrm{Zu}$ Beginn des 21. Jahrhunderts setzte sich der griechisch-französische Komponist Georges Aperghis während seiner Zeit als Kompositionslehrer an der Hochschule der Künste Bern mit diesen drei unterschiedlichen Berner Künstlern auseinander. Er besuchte das Klee-Museum und die Wölfli-Stiftung im Kunstmuseum Bern. Schließlich entstehen zwei abendfüllende Werke, die Wölfli-Kantata (2001-2005) und das Puppentheater Zeugen (UA 2007), in dem Aperghis die im Leben nicht stattgefundene Begegnung von Paul Klee und Robert Walser nachholt. ${ }^{5}$

\subsection{Totale Institutionen in der Schweiz}

Georges Aperghis interessierte sich speziell für das kulturelle Bern der 192oerund 193oer-Jahre, jene Zeit also, während der im Kanton Bern große Dichter und Künstler in Einrichtungen lebten, die der amerikanische Soziologe Erving Goffman »totale Institutionen« nennt:

Ihr allumfassender oder totaler Charakter wird symbolisiert durch Beschränkungen des sozialen Verkehrs mit der Außenwelt sowie der Freizügigkeit, die häufig direkt in die dingliche Anlage eingebaut sind, wie verschlossene Tore, hohe Mauern, Stacheldraht, Felsen, Wasser, Wälder oder Moore. ${ }^{6}$

Goffman zählt zu solchen totalen Institutionen Formen wie Heime, Erziehungsanstalten, Irrenhäuser, Gefängnisse, Kasernen, aber auch Klöster. Die Frage, ob sich die Insassen freiwillig oder gezwungenermaßen in eine totale Institution begeben haben und ob sie zu Recht oder zu Unrecht eingesperrt sind, spielt für Goffman vom System her eine untergeordnete Rolle. Ihn hätte im Falle von Walser der Disput zwischen Psychiatrie und Literaturwissenschaft, ob Walser gesund oder krank war, wenig interessiert. Das macht seinen soziologischen

5 Zu biografischen Bezügen zwischen Klee und Walser siehe Kakinuma: Der Spaziergang in Paul Klees künstlerischem Schaffen. Kakinuma weist nach, dass Klee Fritz Kocher's Aufsätze von Walser gelesen hatte und das Buch 1906 in seinem Tagebuch als »psychologisch sehr ansehnlich « bezeichnete. Ebd., S. 170.

6 Goffman: Asyle, S. 15f. Vgl. auch Kardorff: Goffmans Anregungen für soziologische Handlungsfelder, speziell zur Psychiatrie S. 334-340. 
Ansatz für Walser nicht weniger interessant, denn er beschreibt die »moralische Karriere des Geisteskranken« mit der Unterscheidung von vorklinischer und klinischer Phase so anschaulich, dass man meinen möchte, er hätte Adolf Wölfli und Robert Walser zum Vorbild genommen. ${ }^{7}$

Im jungen Bundesstaat der Schweiz entstehen nach 1848 zahlreiche dieser totalen Institutionen, die das Leben ihrer Insassen gesamthaft oder teilweise regeln und Verstöße gegen die Norm ahnden. Dabei gibt es in der Schweiz auch viele Zwischenstufen in Form von Zwangserziehungsanstalten sowie sogenannte Enthaltungsanstalten für Arbeitsscheue, die zwar als `fauk gelten, aber zu keiner Zuchthaustrafe verurteilt werden konnten, weil sie keine Straftaten begangen haben.

Die Freiheiten, welche die Schweiz 1848 mit der Begründung des demokratischen Bundesstaates errungen hat, werden konterkariert von vielen erzieherischen und domestizierenden Maßnahmen. Der wirtschaftliche Aufschwung verlangt eine disziplinierte Gesellschaft, die sich den utilitaristischen Zielen unterordnet: Erziehung der Willigen auf der einen Seite und Wegsperrung der Arbeitsscheuen, Abseitigen und der sogenannt Irren auf der anderen Seite - das sind die beiden Extreme der damaligen Schweizer Gesellschaft. ${ }^{8}$

Noch $195^{2}$ gibt es in der Schweiz eine ganze Palette totaler Institutionen:

Das neue Strafgesetzbuch verpflichtet die Kantone zur Differenzierung des Strafvollzuges[,] das heisst zur Errichtung von Strafanstalten, Verwahrungsanstalten, Arbeitserziehungsanstalten, Trinkerheilanstalten, Erziehungsanstalten für Kinder und Jugendliche. Die Verurteilten sollen entsprechend ihrer persönlichen Eigenart und ihres Versagens in die passenden Anstaltstypen versorgt werden, um einen optimalen Gesundungserfolg zu erreichen. ${ }^{9}$

In den Künsten und insbesondere in der Musik lässt sich in der Schweizer Geschichte des späten 19. und frühen 20. Jahrhunderts die Herausbildung von zwei Grundtypen beobachten - >Pädagogen $<$ und >Außenseitern $<.^{10}$ Die einen übernehmen oft leitende gesellschaftliche Verantwortung, begründen Schulen, Vereine, kulturelle Projekte und erkennen die Bedeutung der Bildung als Basis für die demokratische Gesellschaft; die anderen finden sich in dieser Gesellschaft nicht zurecht, scheitern, benehmen sich auffällig, werden asozial oder

7 Goffman: Asyle, S. 125-167.

8 Bei den Gebäudehüllen dieser totalen Institutionen gibt es erstaunliche Kontinuitäten: Aus den Klöstern werden Irrenanstalten, aus den Tollhäusern Gefängnisse, aus Schlössern Zuchthäuser und Internate.

9 Maurer: Was will die Arbeitserziehungsanstalt Uitikon?, S. 319.

10 Vgl. Brotbeck: Zum Schweizerischen in der Schweizer Musikgeschichte des 20.Jahrhunderts. 
delinquent und landen häufig als Insassen in der einen oder anderen totalen Institution, wo sie oftmals zu einem Forschungsgegenstand werden, den man auch in Vorlesungen präsentiert. ${ }^{11}$ Wer aus einer totalen Institution entlassen wird, entkommt ihr auch außerhalb der Anstaltsmauern nie ganz, denn die entsprechenden Erfahrungen prägen das weitere Leben, was sich zum Beispiel im Werk der Schweizer Schriftsteller Carl Albert Loosli ${ }^{12}$ oder Friedrich Glauser eindrücklich beobachten lässt. ${ }^{13}$

\section{7·3 >Schizophrenelisgärtli<}

Im Zusammenhang mit Aperghis' Beschäftigung mit Wölfli, Walser und Klee ist es brisant, dass sich auch der berühmte Bauhausmeister nach seiner Rückkehr in die Schweiz mit latenten Vorwürfen konfrontiert sah, psychisch krank zu sein oder es werden zu können. Zwar drohte Klee dabei nicht die Einlieferung in eine totale Institution, aber er musste doch befürchten, dass sein Einbürgerungsgesuch abgelehnt würde.

Die NS-Kulturpolitik warf dabei ihre Schatten auf die Schweiz. 1933 wurde Klee von seiner Tätigkeit als Professor an der Kunstakademie Düsseldorf freigestellt; der Kunstschriftleiter des Völkischen Beobachters, Robert Scholz, griff ihn in der Zeitschrift Deutsche Kulturwacht, Blätter des Kampfbundes für deutsche Kultur frontal an. Klee gehörte für Scholz zu jenen »sozial kranken« Künstlern, die im verhassten Frankreich - und hier vor allem in der »morbiden Atmosphäre« von Paris - angesteckt »nur Geistig-Krankes schaffen«:

Und daß man Paul Klee einmal als großen Künstler ansehen konnte, wird für künftige Generationen eines der deutlichsten Exempel des völligen geistigen Verfalls der individualistischen Kulturepoche sein. Dinge, die nur die Lachmuskeln reizen konnten, wie das irrsinnige, kindische Geschmiere eines Klee, wurden als der Gipfelpunkt schöpferischer Sensibilität angepriesen. ${ }^{14}$

11 Heinz Holliger erzählte während der Holliger-Walser-Woche 1996 in Biel, dass sein Vater während des Medizinstudiums an einer medizinischen Vorführung von Adolf Wölfli teilgenommen hatte.

12 Loosli: Anstaltsleben.

13 Das in totalen Institutionen der Schweiz im 2o. Jahrhundert verübte Unrecht wurde im Auftrag des Schweizer Bundesrats von der Unabhängigen Expertenkommission (UEK) Administrative Versorgung bis 1981 untersucht. Die Arbeit der Kommission wurde 2019 mit einem Schlussbericht beendet, dessen Quellenband das ganze Ausmaß des Repressions- und Verwahrungssystems belegt. Vgl. Huonker et al.: ... so wird man in ein Loch geworfen.

14 Scholz: Kunstgötzen stürzen, S. $5^{2 f}$. 
Die von Robert Scholz formulierten Vorwürfe des Kindischen, ${ }^{15}$ der Hirngespinste, des Geisteskranken, des Großstädtischen und der Orientierung an der Kunst fremder Völker, ja der >Negerkunst< klingen auch in der Schweizer Presse an. So schreibt ein gewisser »h.gr.« 1935 in der Neuen Zürcher Zeitung zur Klee-Ausstellung in der Kunsthalle Basel: »[W]as uns vor allem skeptisch stimmt, ist das deutliche Anknüpfen an Kinderzeichnungen und Verwandtes. Wenn ein über fünfzigjähriger Mann sich von solchen Arbeiten inspirieren läßt, so können wir darin nicht etwas absolut Positives sehen. «16

1940, nach einer großen Ausstellung im Kunsthaus Zürich zu seinem Schaffen von 1935 bis 1940, konnte sich Paul Klee eigentlich versichert sein, in der Schweiz seinem Wert entsprechend wahrgenommen zu werden, zumal Max Bill zu diesem Anlass einen denkwürdigen Aufsatz in zwei Teilen in der Neuen Zürcher Zeitung (NZZ) publiziert hatte. ${ }^{17}$ Den Bericht zur Vernissage dieser Ausstellung verfasste Jakob Welti, der konservative Kunstkritiker der NZZ, noch einigermaßen neutral. Er charakterisiert Klee als »Großstadtmensch mit einem überreichen, lastenden Bildungsgut «, was sich in den zahlreichen Stilen zeige, an die er sich anlehne: »Höhlenzeichnungen [...], Negerplastik, inneramerikanische Indianerkunst, Alt-China, Japan, [...] frühchristlich, koptisch, byzantinisch, romanisch ${ }^{18}$

Möglicherweise waren für Welti gerade Max Bills Huldigungen und die allgemein positive Aufnahme der Klee-Ausstellung in der Schweizer Presse ${ }^{19}$ des Guten zu viel. Er nutzte die Folgeausstellung im Zürcher Kunsthaus zu acht Aargauer Malern und Plastikern, um Klee doch noch an jenen Ort zu kalauern, wohin er seiner Meinung nach gehörte, nämlich in psychiatrische Obhut:

Nach der interessanten März-Exkursion in das eigenartige, vielen Besuchern zu hoch gelegene Schizophrenelisgärtli ${ }^{20}$ Paul Klees, befindet man sich wieder in den klimatisch und optisch vertrauteren Gefilden der schweizerischen Mittellandsmalerei. ${ }^{21}$

\footnotetext{
$15 \mathrm{Zu}$ Klee und seiner Nähe zu Kinderzeichnungen siehe Werckmeister: Klees `kindliche Kunst.

16 h.gr:: Kunst in Basel.

17 Bill: Zur Ausstellung Paul Klee im Kunsthaus Zürich I und II.

18 Welti: Aus dem Zürcher Kunsthaus, 19.02.1940.

19 Frey: Chronologische Biographie (1933-1941), S. 122f.

20 Das Vrenelisgärtli ist eine vielbestiegene Bergspitze der Glarner Alpen, um die sich viele Vreneli-Sagen ranken.

21 Welti: Aus dem Zürcher Kunsthaus, 30.03.1940.
} 
Paul Klee muss diese Einschätzung enorm geärgert haben. Er beauftragte seinen Anwalt, Welti wegen Rufschädigung einzuklagen und eine Richtigstellung in der Zeitung zu fordern, sah dann aber davon ab: »Um in der Öffentlichkeit und bei den Behörden kein weiteres Aufsehen zu erregen, verzichtete er jedoch auf eine Gegendarstellung. ${ }^{22}$

In den Befragungsprotokollen des Einbürgerungsverfahrens durch die Polizei ist folgende Bemerkung zu Klee protokolliert: »Litt vor Jahren wahrscheinlich an einer neurotischen Blutgefässtörung. Ist jedoch heute nicht mehr in ärztlicher Behandlung und fühlt sich wieder hergestellt. ${ }^{23} \mathrm{Nach}$ Walther Fuchs hat die Polizei unter Neurose wahrscheinlich eine ausschließlich psychische Erkrankung verstanden. Das würde auch den Unmut Klees über den Kalauer >Schizophrenelisgärtli< erklären.

\subsection{Zwischenstück: Paul Klee und der Polizist}

Mit den von Klee geschaffenen Kasperlepuppen Selbstporträt und Polizist könnte man die peinliche Befragung nachspielen, der Paul Klee während seines Einbürgerungsverfahrens 1939 ausgesetzt gewesen sein musste. Davon zeugt ein sogenannter >Geheimbericht< in französischer Sprache, den Meta La Roche einsehen konnte und aus dem sie 1957 im Sankt Galler Tagblatt längere Abschnitte zitiert. ${ }^{24}$ Verfasst wurde er gemäß La Roche von einem Wachtmeister der Berner Kantonspolizei, der sich in einem etwas gestelzten, zuweilen fehlerbehafteten Französisch ausdrückt. Diese Auszüge werden hier erstmals in revidiertem Französisch publiziert. ${ }^{25}$ Da die Originaldokumente

22 Fuchs: Paul Klee und seine Krankheit revisited, S. 65.

23 Zitiert nach ebd., S. 64. Die Schreibweise >Blutgefässtörung«, die Fuchs zu »Blutgefäs[s] störung « ergänzt, entspricht der damaligen schweizerischen Orthografie-Regel, eine Aneinanderreihung von drei identischen Buchstaben zu vermeiden.

24 La Roche: Die Schweiz, Jean Arp, Paul Klee und ein geheimer Polizeirapport. Aus diesem Bericht wird meist nur der eine Satz zitiert, Klees Werk sei »eine Beleidigung gegen die wirkliche Kunst und eine Verschlechterung des guten Geschmacks«. Als Beispiel sei der Wikipedia-Artikel zu Paul Klee angeführt. Stefan Frey hat 1990 im Katalog zur KleeAusstellung im Kunstmuseum Bern erstmals das dreistufige Verfahren des Berichtes hervorgehoben. Vgl. Frey: Chronologische Biographie (1933-1941), S. 11gf.

25 Gewisse Mängel könnten sich auch beim Abschreiben oder beim Setzen des Textes eingeschlichen haben. Hier werden offensichtliche Akzentfehler stillschweigend korrigiert; ergänzte Auslassungen und Veränderungen sind mit eckigen Klammern bzw. Anmerkungen angezeigt. Die Satzzeichen sind von der im Sankt Galler Tagblatt verwendeten deutschen Setzung übernommen. 
1960 zerstört wurden, ${ }^{26}$ ist der Artikel von Meta La Roche bis heute die einzige Quelle zu diesen Polizeirapporten. ${ }^{27}$ Sie sind ein einmaliges Dokument insbesondere im Kontext von Adolf Wölfli und Robert Walser: Wölfli wurde schon zu Lebzeiten von den Ärzten wegen seines neuartigen Schaffens zum Künstler erklärt; bei Walser wird noch im 21. Jahrhundert von Psychiatern aufgrund seines Schreibens eine Katatonie diagnostiziert (vgl. Einleitung); im Falle von Paul Klee versucht der Wachtmeister aus seinem Schaffen und Verhalten - in ähnlicher Weise wie die Psychiater im Falle Walsers - die Veranlagung zu einer psychischen Krankheit zu suggerieren und mit einem seiner Bilder eine antischweizerische Gesinnungsart zu beweisen. Der >Geheimbericht bestätigt exemplarisch die von Goffman beschriebenen Verfahren der totalen Institution. In Goffmans Personalkategorien totaler Institutionen wäre der Wachtmeister ein Vertreter »der niedrigsten Personalränge. Es ist ein besonderes Merkmal dieser Gruppe, daß ihre Mitglieder meist in langfristigen Beschäftigungsverhältnissen stehen und daher Träger der Tradition sind «. ${ }^{28}$ Allerdings hält sich dieser Wachtmeister nicht an die Rollendifferenzierung und greift ins Kompetenzgebiet ein, das höheren Personalrängen wie Ärzten vorbehalten ist: Geradezu megaloman spielt er sich nicht nur als Psychiater, sondern auch als Jurist und Kunstsachverständiger auf.

Der Bericht des namentlich nicht genannten Wachtmeisters wurde in drei Tranchen verfasst. Über das Umfeld der drei Berichte kann nur gerätselt werden; die thematische Gliederung könnte auf eine Form von Interaktion mit der eidgenössischen Einbürgerungsbehörde hinweisen: Im ersten Bericht die Desavouierung der Kunstverständigen, im zweiten die Warnung vor Klees vermuteter Geisteskrankheit und im dritten der >Beweis` dafür, dass Klee ein Nestbeschmutzer der Schweiz sei.

26 Vgl. Kehrli: Weshalb Paul Klees Wunsch, als Schweizer zu sterben, nicht erfüllt werden konnte, S. 10.

27 Meist dient dieser >Geheimbericht` dazu, das schweizerische Einbürgerungsverfahren von Paul Klee zu >skandalisieren < und Verzögerungsversuche zu belegen. Otto Jakob Kehrli wies allerdings schon 1963 minutiös nach, dass dies faktisch falsch ist und das Verfahren nach den Vorschriften des im Mai 1933 mit dem >Dritten Reichく vereinbarten, skandalös restriktiven Einbürgerungsgesetzes der Eidgenossenschaft ablief. Vgl. Kehrli: Weshalb Paul Klees Wunsch, als Schweizer zu sterben, nicht erfüllt werden konnte, S. 10. Einzig mit der Bewertung, die stadträtliche Einbürgerungskommission habe allein »im Interesse des Gesuchstellers « (S. 11) nach der eidgenössischen Bewilligung vom 19. Dezember 1939 noch zweimal weitere Erklärungen gewünscht (und damit die Einbürgerung nochmals um zwei Monate verzögert), beurteilt Kehrli als ehemaliger Oberrichter des Kantons Bern die administrativen und politischen Entscheidungsträger wohl etwas zu wohlwollend. 
Am 31. Oktober 1939 stellt der Wachtmeister einleitend fest, dass Klee, seine Schwester und ihr Vater »des personnes très susceptibles et pénibles ${ }^{29}$ seien.

Dann legt der Polizist dem Bericht Hans Christian Andersens Märchen Des Kaisers neue Kleider bei, um die Behörden darauf aufmerksam zu machen, dass die einflussreichen Bewunderer Klees dessen Bilder nur deshalb als Kunst betrachten würden, weil es ihnen vorgemacht werde.

Le candidat, tête de l'intellectuel classique, semble être complètement plongé dans un monde spirituel. Il prétend être radicalement expression[n]iste, ne se laisser influencer par personne et donnant ainsi, par son art à lui, le meilleur de son individualité. Les admirateurs de Klee, personnalités très influentes, prétendent comprendre ou saisir la révélation de son être intime. Dès lors, ne serait-il pas utile de chercher à caractériser le candidat par l'hommage-même que lui rend l'auteur de l'ouvrage »Paul Klee«? dont un extrait avec illustration [est] joint au présent rapport.

Ci-joint une autre copie, du conte: »Les habits neufs du grand-duc« ou »Des Kaisers neues Kleid«. Or, ce récit ne serait-il pas [fait] pour illustrer les admirateurs des prétendues chef-d'œuvres du prof. Klee? Dans ce récit figure une image représentant le grand-duc auprès des tisserands et cherchant sur leur métier vide, au moyen de sa loupe, la merveilleuse étoffe commandée par le ministre du grand-duc. Bien que n'y voyant goutte, mais, ${ }^{30}$ pour éviter d'être taxé d'imbécile - de ne rien voir - il préfère avouer l'avoir vue et, par[-]dessus tout, merveilleuse! Une parallèle ne pourrait-elle pas être tirée ici entre les admirateurs du prof. Klee et ce bon vieux ministre du grand-duc, voire tous ceux qui après lui prétendront ${ }^{31}$ avoir vu cette étoffe inexistante?

Es folgt der Abschnitt mit dem erwähnten oft zitierten Satz, bei dem der Wachtmeister vorgibt, die Meinung sehr bekannter Maler weiterzugeben.

Des peintres bien connus de chez nous estiment que cette »Neue Richtung « fantaisiste de Klee leur serait funeste si, bénéficiant de la protection de certaines personnes compétentes, ce genre de peinture devait prendre pied dans notre pays. Ce serait une insulte à l'adresse du vrai art de la peinture, un avilissement du bon goût et des idées saines de la population en général.

Vier Tage später, am 4. November 1939, verfasst der Berner Polizist seinen zweiten Bericht. Er hatte verschiedene Persönlichkeiten befragt, aber von keinem die von ihm gewünschte Antwort bekommen, man solle Klee die Einbürgerung verweigern. Und man kann nur vermuten, dass die angefragten

29 Alle Zitate aus La Roche: Die Schweiz, Jean Arp, Paul Klee und ein geheimer Polizeirapport, [S. 11].

30 Die Satzkonstruktion müsste lauten »bien que ... et« und nicht »bien que ... mais«.

$31 \quad$ Im Original »prétendiront«. 
Akademiker dem Wachtmeister nicht den gebührenden Respekt zukommen ließen, denn er wirkt verletzt und beleidigt. Auch dieses Verhalten von Vertretern totaler Institutionen hat Erving Goffman präzise beschrieben:

Ein beleidigter Empfänger kann gegen denjenigen, der sich nicht genügend ehrerbietig verhält, einschreiten, aber für gewöhnlich muß er die wirklichen Gründe für solche korrigierenden Maßnahmen verheimlichen. ${ }^{32}$

Der Wachtmeister konstruiert mit dem Verweis auf den die Nationalitäten wechselnden und zum Schluss in »Paris (!)« endenden Kandinsky im Stil von Robert Scholz eine internationale Szene von Intellektuellen und Spekulanten, darunter selbstverständlich viele Juden, welche sich die Bilder des scheinbar bescheiden und zurückgezogen lebenden Künstlers bloß als Kapitalanlage anschaffen:

Les références indiquées par Klee dans sa demande en naturalisation, celles soi-disant versées dans son art, le recommandent naturellement au mie[u]x. Elles estiment que ses dessins, quoiqu'en partie extravagants, sont malgré tout le produit d'un intellectuel exceptionnel. On s'arrêterait toutefois plutôt au superbe et extraordinaire assemblage de couleurs donné par sa peinture.

Le Dr. phil. X., camarade d'école de Klee, croit que ce genre d'art aura une continuation parce qu'étant quelque chose d'entièrement nouveau. Le Dr. Y. par contre prétend que Klee ne fera pas école et qu'ainsi il ne gênera pas aux peintres du pays. Ce dernier avait constaté, dans ses nombreux voyages en Allemagne, avant l'arrivée au pouvoir du hitlérisisme [sic], que les extravagances apportées dans l'art en général par le »Bauhaus« de Dessau, allaient à l'encontre d'une réaction certaine.

Ce »Bauhaus« en effet était devenu un centre intellectuel ultra-moderne et où Klee y trouvait son semblable: le maître peintre artiste russe Kandinsky, devenu Allemand et aujourd'hui Français domicilié à Paris (!).

Le Dr. Z., le Dr. et prof. XY. et autres estiment que, malgré ses bizarreries, Klee ne démérite pas la naturalisation suisse. On ne craindrait nullement chez lui une aliénation mentale, parce que grand génie resté modeste.

Cette prétendu[e] modestie ne serait-elle pas plutôt de trompeuse apparence? Car ne verrait-on pas Klee justement rester soi-disant modeste et retiré du brouhaha de ce monde sachant fort bien que d'autres pour lui se remuent, l'élèvent jusqu'aux nues et ainsi lui font la réclame, voir les éloges grandiloquents à son adresse par Grohmann, l'auteur de l'ouvrage »Paul Klee« et ceux de H. v. Wedderkop dans »Junge Kunst«, Band 13 etc. Ceci sans oublier la presse.

Cette réclame d'autre part, ne serait-elle pas faite à dessein par certains intéressés trouvant en Klee l'objet approprié à leur fin matérielle? L'inédit, le singulièrement surprenant que Klee leur fournit aujourd'hui, cela ne pourrait-il pas un jour les amener au comble de leur cupidité? C'est-à-dire, ces intéressés directs ne trieraient et ne collectionneraient-ils pas, du vivant de Klee, ses 
peintures en vue d'un futur coup de commerce fabuleux? Car le moment venu, ces gens-là n'auraient-ils pas alors tous les moyens à disposition pour exceller de plus encore la valeur artistique de leurs prétendus chef-d'œuvres et ainsi en multiplier la valeur vénale? et surtout quand, au cas particulier, on apprend que ce genre d'intellectuels profiteurs se recrutent dans le monde juif!

Nach solcher Vorbereitung kann der Wachtmeister nun zum eigentlichen Punkt kommen, nämlich zu Klees Prädisposition für eine Geisteskrankheit:

Dans ce cas, Klee n'en serait-il pas que le jouet? Un prétendu esprit surnaturel, imaginé, encouragé et ex[hi]bé pour les besoins de la cause? c'est-à-dire poussé à des extravagances toujours plus grandes, au profit d'autrui? Dès lors, n'y en aurait-il pas assez pour lui faire perdre un jour la raison? Bien que [de] grande intelligence et appar[e]mment modeste ne se serait-il pas cependant laissé bourrer le crâne? Tous ceux qui le connaissent très bien avouent qu'en effet il a l'air d'un visionnaire. Et certains le voient friser l'aliénation mentale. Klee prétend de rien avoir [à faire] ${ }^{33}$ avec l'anthroposophie bien que les adeptes de cette association s'intéresseraient à son art.

Ob dieser Bericht bei seinem Vorgesetzten noch immer nicht auf die erwartete Resonanz stieß? Jedenfalls holt der Wachtmeister am 9. November 1939 zum finalen Schlag aus: Anhand eines Bildes von Klee weist er nach, dass dieser während seiner Karriere in Deutschland nicht nur keinerlei Anzeichen machte, in die Schweiz zurückkehren zu wollen, sondern seine alte Heimat mit Kühen, die unter einer Schweizer Fahne dumm in die Landschaft glotzten, sogar noch verspottete. Und nun wolle er sich plötzlich an die schweizerischen Sitten angepasst haben:

Le changement du régime gouvernemental allemand l'ayant contraint de revenir en Suisse, dans sa »alte Heimat« comme il l'appelle aujourd'hui, ne serait-ce pas que notre pays [l']aura été trouvé, à ce moment-là, de nouveau assez bon pour le recevoir?

Klee dit bien s'être rendu en Allemagne pour ses études et afin d'y développer son art efficacement, la Suisse ne possédant pas les institutions à cet effet. Cependant, ne serait-il pas resté si longtemps en Allemagne précisément parce que là-bas il se sentait chez lui? fait à d'autres mœurs et à une culture soidisant plus élevée que celle de chez nous? Voir la signification de son tableau »Schweizer Landschaft« qu'il donnait en 1919 et dont [une] copie au dossier concernant sa naturalisation.

Ce tableau ne parlerait-il pas tout simplement, sans ambages, de ce que certains se plaisent à représenter sous la désignation de »Kuhschweizer«? Cet[te] énigme, s'il en existe, Klee explique en prétendant qu'il s'agit, en l'occurrence, 
d'un quelconque ${ }^{34}$ paysage du Simmental! Etait-ce alors nécessaire d'y faire dominer des vaches avec l'air si »bête« et sous le sceau de notre emblème national?

De plus amples renseignements à ce sujet ne peuvent être obtenus de Klee, son art devant être comme une sorte de prédiction mystérieuse: selon »Neue Kunst « ou plutôt »Junge Kunst«: da er ausgesprochen individualistisch bestimmt ist, ein Einzelfall, zu dessen Erkenntnis kein anderer Weg führt als das Mitfühlen.

Or, ce »Mitfühlen« non mystique mais logique, ne nous permettrait-il pas justement de comprendre que par cette »Schweizer Landschaft « Klee aura tout bonnement voulu se moquer de nous? Et aujourd'hui, de par la force des choses, il se serait réassimilé à nos mœurs?

Dieser Bericht hatte zwar auf das Einbürgerungsverfahren keinerlei Einfluss, aber für den bereits schwer erkrankten Klee muss diese Episode mit dem der Zeitpropaganda folgenden und absurde Schlüsse ziehenden Polizisten belastend gewesen sein, und sie erklärt wohl auch, weshalb der Künstler so empört auf den Kalauer >Schizophrenelisgärtli< reagierte.

\subsection{Der Komponist Georges Aperghis}

Als Georges Aperghis, der von 2003 bis 2008 zusammen mit der französischen Schlagzeugerin und Performerin Françoise Rivalland an der Hochschule der Künste Bern die Klasse für Théâtre musical aufbaute, angestellt hätte werden sollen, ergab sich ein administratives Problem: Aperghis verfügte über keine Schulzeugnisse. Es bedurfte einiger Erklärungen, um die Administration des Kantons Bern davon zu überzeugen, einen Mann ohne formale Schul- und Studienabschlüsse als Hochschuldozent anzustellen.

Tatsächlich ist Georges Aperghis ${ }^{35}$ ein eingefleischter Autodidakt. Die Schule sei die schlimmste Zeit seines Lebens gewesen; er habe da Partituren studiert, statt den Lehrern zuzuhören. Den ersten Kompositionsunterricht in Griechenland bezieht er nicht in einer Musikschule, sondern bei Freunden von Freunden seiner Eltern. Die Mutter ist Malerin, der Vater Bildhauer; es ist schließlich der Vater, der dem damals 18-jährigen Sohn rät, die Zelte in

34 Im Original: »quelquonque«.

35 Georges Aperghis ist musik- und theaterwissenschaftlich noch wenig erforscht. Matthias Rebstock publizierte mit >Ça devient du théâtre, mais ça vient de la musique einen Text in englischer Sprache. In Frankreich beschäftigt sich Jean-François Trubert mit Aperghis, vgl. Trubert: Fragments de geste dans le théâtre musical expérimental sowie Donin/Trubert: Georges Aperghis. 
Griechenland abzubrechen und in Paris das zu werden, was er schon immer werden wollte: Musiker. ${ }^{36}$

In Paris folgt dann eine fast sieben Jahre dauernde Inkubationsphase, während der sich Aperghis mit Gelegenheitsjobs wie Kino-Bewachen, Notenkopieren oder als Pianist in einer Tanzkapelle durchschlägt. Daneben stürzt er sich auf alles, was er an zeitgenössischer Musik hören kann. Er kommt in Kontakt mit der von Pierre Boulez geleiteten Konzertreihe »Domaine musical« und freundet sich mit seinem ebenfalls in Paris lebenden Landsmann Iannis Xenakis an. Allerdings fühlt sich Aperghis von keiner der zahlreichen musikalischen Strömungen wirklich angezogen; wohl auch deshalb mag er sich zu keiner strukturierten Ausbildung entschließen. Er bildet sich autodidaktisch weiter, indem er - keineswegs nur zeitgenössische - Partituren studiert.

Bei einer Veranstaltung der kommunistischen Partei lernt er die Schauspielerin Édith Scob kennen, die beiden heiraten $1965 .{ }^{37}$ Über sie kommt er zu Aufträgen für die Hörspielabteilung von Radio France, wo er Gebrauchsmusik in unterschiedlichsten Stilrichtungen schreibt. ${ }^{38}$ Auch sein Weg zum Szenischen, Theatralischen, Sprachlichen wird stark von Édith Scob inspiriert und unterstützt. In vielen seiner frühen Stücke spielt sie als Schauspielerin und Sprecherin mit. Mit ihr und dem Schlagzeuger und Komponisten Jean-Pierre Drouet wagt er ab 1976 das ATEM-Projekt, das viel von sich reden macht. ATEM steht für »L'atelier théâtre et musique« und wird von 1976 bis 1991 von der Gemeinde Bagnolet finanziert, einer Pariser Vorortsgemeinde, die bis 2014 Teil der sogenannten >ceinture rouge war, also jenem Gürtel um Paris mit kommunistisch dominierten Gemeinderäten. ATEM durchmischte »künstlerische Produktion, Theater- und Musikpädagogik und soziales Engagement. Ästhetische Experimente wurden in den Dienst der Begegnung « gestellt, verwandt den $»$ heutigen education-Projekten ${ }^{39}$

Von 1976 bis 1988 haben sich 335 Menschen am ATEM-Projekt von Aperghis beteiligt. Wenn man diese Liste durchgeht, begegnen einem die führenden Vertreterinnen und Vertreter der zeitgenössischen Musik neben zahlreichen unbekannten Personen und Menschen, die nur mit Vornamen genannt sind, darunter wohl auch sogenannte SDF (>sans domicile fixe $<)^{40}$

36 Vgl. France Musique: Les grands entretiens. Georges Aperghis (1/5).

37 Vgl. Guyard: Mort d'Édith Scob.

38 Vgl. France Musique: Les grands entretiens. Georges Aperghis (2/5).

39 Dick: Zwischen Konversation und Urlaut, S. 240.

40 Vgl. »Ont collaboré à l'ATEM (de 1976 à 1988)«. Gindt: Georges Aperghis, S. 263-265. 
Von den vielen Projekten, die unter dem Schirm von ATEM entstanden, gibt es nur vereinzelt Partituren oder Aufzeichnungen, weil Aperghis weitgehend mit Laien, interdisziplinär und mit jenem Instrument arbeitete, das uns am nächsten ist, dem Körper:

[U]ne troupe de comédiens est venue repérer les lieux, est venue expliquer qu'ici à Bagnolet, on peut faire du théâtre, de la musique, autrement, sans forcément écrire les notes sur un papier à cinq lignes, sans toujours utiliser les instruments à cordes, à anches, à pistons. On fera, ont-ils dit, avec ce qui existe, tous les jours, en répétant et avec le plus fou des outils: le corps. ${ }^{41}$

In den 20 Jahren der ATEM-Zeit - nach Bagnolet wurde das Projekt von 1991 bis 1997 in der Pariser Vorortsgemeinde Nanterre-Amandiers weitergeführt entwickelt Aperghis die Grundprinzipien seines kompositorischen Denkens und Handelns:

Deux idées président au travail de création et de gestion à l'ATEM. La seconde est la tendresse. La première, le détournement. Et il n'y a pas de coupure quant à leur application entre le travail de création et celui de l'intendance. Détourner les objets, les idées, les sons, constitue pour nous l'essentiel de nos désirs artistiques. Rendre le social intime, le sonore visuel, le concert théâtral, les mots musique, le sentimental comique, nous semble un jeu vital dans la polémique que nous engageons contre le goût du jour. Ceci se retrouve dans la reproduction de spectacles que nous présentons et qui peuvent dérouter par le déplacement des valeurs et des idées. Ainsi l'ATEM fonctionne comme une sorte de commando de détournement généralisé. Quant à la tendresse, c'est celle que nous éprouvons pour les objets, les choses et les sons abandonnés par tous, réputés sans valeur, les chutes, les mauvaises idées, les concepts contre lesquels on a dit tout le mal possible et qui souvent, vus sous un autre angle, reprennent vie et inspiration. ${ }^{42}$

In dieser Spannung von $>$ Détournement $<$ und $>$ Tendresse $<$ arbeitet Aperghis bis heute; sie bestimmt auch seine Arbeit zu Walser, bei dessen Schriften ihn das >Détournement $<$ - die Ablenkung, Umdrehung ebenso wie die Entführung besonders fasziniert. >Détournement< und >Tendresse $<$ - Liebkosung, Weichheit, Zärtlichkeit, Nachsicht - erklären auch, weshalb die Werke von Aperghis nie politisch im Sinne von Maurizio Kagel sind: Das Abgrenzende, Aggressive, Anklagende, zuweilen auch Denunziatorische, das politisches Musiktheater nach Kagel ausmacht, ist Aperghis fremd. Diese `Zärtlichkeit< hat auch eine

41 Ebd., S. 43.

42 Aperghis zitiert nach ebd., S. 65. 
Qualität von ATEM ausgemacht: Da wurden nicht Ideologien zu Markte getragen, sondern mit Leib und Seele ein neuartiges, fantastisches Theater aufgebaut, das weder mit dem traditionellen Dialogtheater noch mit traditionellen Kompositionen etwas zu tun hatte.

Aperghis entwickelte für die Mitwirkenden eine eigene $>$ Sprache $\prec$. Diese an Lautpoesie erinnernden Ketten von Silben und sprachähnlichen Strukturen, die ihren Bedeutungsgehalt nur vorgaukeln, prägen bis heute Aperghis' theatralische Werke. Das wirkt manchmal wie eine Improvisation oder als Einladung an die Interpretinnen und Interpreten zu improvisieren. Das ist aber nicht der Fall:

Moi, je n'improvise pas du tout en amont de l'écriture. Par contre, avec les années, j'ai accumulé un fond de matrices, on peut les appeler des fichiers, des sortes de réservoirs de techniques de composition, des matrices de permutation, ou des structures polyphoniques par exemple. Ce sont des brouillons qui étaient pour une pièce mais qui finalement pouvaient donner naissance à plusieurs variantes, et donc à des musiques complètement différentes. J'en ai gardé énormément, et je travaille beaucoup à partir d'eux. C'est-à-dire que lorsque j'ai une idée, pour essayer de la construire et de la mettre sur le papier, je passe par différents anciens brouillons - ou fichiers ou ... je ne sais pas comment les appeler - pour voir ce qu'ils me proposent comme solution. Finalement, je trouve une autre solution qui est une variante ou une espèce d'annexe de différentes choses que j'ai gardées. Mais ce qui m'intéresse, c'est d'arriver à la surprise, d'être surpris par ce qui peut en sortir, par ce que je n’avais pas du tout imaginé au départ. ${ }^{43}$

Diese >matrices< bilden ein Reservoir von Blättern und Skizzen mit Wort- und Sprachpermutationen, Transformationen und Verfremdungen von bekannten Wörtern, auf die Aperghis beim Komponieren wie auf Motivtabellen zurückgreift. Wir begegnen den Spuren dieser >matrices` auch wieder bei seinen Werken zu Wölfli und Walser. Im Falle von Wölfli hat er dessen Texte in Silben zerstückelt und daraus eine eigene >matrice gebildet. Bei Walser hat er die Originaltexte weitgehend belassen, sie aber mit >matrices` überlagert und umrankt. 


\title{
7.6 Wölfli-Kantata
}

Bis zum 150. Geburtstag von Adolf Wölfli 2014 war Georges Aperghis nach Johannes Harneit (vgl. Kap. 9.12) erst der zweite Walser-Vertoner, der sich auch mit Adolf Wölfli kompositorisch beschäftigt hat. ${ }^{44}$ Für einen des Deutschen unkundigen Komponisten ist dies einigermaßen auffällig.

Die Wölfli-Kantata (2001-2005) hat Aperghis für die Neuen Vocalsolisten Stuttgart und das SWR Vokalensemble geschrieben, beides international führende Ensembles. Die Neuen Vocalsolisten sind ein Solistenensemble von sieben Mitgliedern, darunter der Countertenor Daniel Gloger, der in der Kantata von Aperghis an wichtigen Schaltstellen eine führende Rolle übernimmt. Aperghis wählte drei hohe Stimmen (Koloratursopran, Mezzosopran und Countertenor) und drei tiefe Stimmen (Tenor, Bariton, Bass). Das SWR Vokalensemble gilt als einer der wichtigsten Rundfunkchöre Europas, der aus 36 Berufssängerinnen und -sängern besteht. Wegen seiner regelmäßigen Vergabe von Kompositionsaufträgen ist seine Besetzung in der zeitgenössischen Musik inzwischen zum Standard geworden. Auch Aperghis übernimmt alle 36 Chor-Positionen in der Kantata. Das Werk besteht aus fünf Sätzen, davon sind zwei für das Solistenensemble, zwei für den Chor und der letzte Satz für beide Ensembles zusammen komponiert:

\author{
Petrrohl, pour 6 solistes vocaux \\ Die Stellung der Zahlen, pour chœur mixte \\ Vittriool, pour 6 solistes vocaux \\ Trauer-Marsch, pour chœur mixte \\ Von der Wiege bis zum Graab, pour solistes et chœur ${ }^{45}$
}

Georges Aperghis hat sich nicht mit der Vertonungsgeschichte von Adolf Wölfli beschäftigt, und doch - oder gerade deswegen - unterscheidet sich seine Wölfli-Kantata markant von anderen musikalischen Auseinandersetzungen mit dem berühmten Waldau-Patienten. Der Hauptgrund dafür liegt in der

44 Zu diesem Jubiläum hat der Verein Wölfli \& Musik Werkaufträge auch an die WalserKomponistinnen und Komponisten Daniel Glaus, Christian Henking, Ezko Kikoutchi, Roland Moser und Annette Schmucki erteilt. Vgl. Wölfli \& Musik: Kompositionen zu Wölfli.

45 An der Komposition arbeitete Aperghis über vier Jahre: Petrrohl ist mit »juin 2001« datiert; Von der Wiege bis zum Graab trägt die Enddatierung »23 juillet 2005«. 
Tatsache begründet, dass Aperghis Wölflis Deutsch nicht >versteht $<$ und seine Sprache nur als Klang wahrnimmt. Die dialektalen Einflüsse, die eigenwillige Orthografie und die überraschenden Satzkonstruktionen fallen Aperghis wegen mangelnder Deutschkenntnisse nicht weiter auf. Auch die zahllosen Reime von Wölfli sind für Aperghis ein rein sprachklangliches Phänomen, das den `matrices<, mit denen er seine Sprachkompositionen gestaltet, eng verwandt ist.

Bei vielen Komponistinnen und Komponisten führt gerade die Radikalität, mit der Wölfli den Reim gegen Inhaltliches durchsetzt und Wörter sogar deformiert, um sie dem Reim anzupassen, zu volksliedhaften oder tanzähnlichen Werken. Im Vordergrund stehen das Schiefe, Verlotterte und die unfreiwillig komischen Megalomanien Wölflis.

Oft wird auch - mit Wölflis Bildern und Texten - seine Biografie vertont. Am weitesten ist hier der dänische Komponist Per Nørgård (“1932) gegangen, der 1979 im Louisiana Museum of Modern Art in Humlebæk (Dänemark) Wölflis Werk begegnete ${ }^{46}$ und sich in der Folge mehrere Jahre mit ihm beschäftigte; seine Oper Der göttliche Tivoli ${ }^{47}$ (1982) ist das zentrale Werk dieser Auseinandersetzung. ${ }^{48}$

Vom virtuosen Theatermann Aperghis hätte man bei Wölfli eigentlich ein mehrfach überlagertes Théâtre musical erwarten können. In diesem Fall aber beschränkt er sich auf rein konzertante Mittel. Weder Wölflis Biografie noch seine Bilder und Kompositionen, aus denen andere Komponistinnen und Komponisten Melodien und Rhythmen ableiteten, sind von Bedeutung. Auch Wölflis Schizophrenie interessiert ihn kaum. Der entscheidende Anstoß zur Komposition war ein Besuch im Wölfli-Archiv des Kunstmuseums Bern, wo der damalige Kurator Daniel Baumann dem Komponisten die Zeichnungen

46 Outsiders. An Art without Precedent or Tradition, Wanderausstellung, 1979/8o, Louisiana Museum, Humlebæk, 22. September bis 11. November 1979. Zur Ausstellung vgl. das Plakat unter Litografier: Adolf Wölfli, zu Nørgårds Begegnung mit Wölfli vgl. Hansen: Per Nørgård. Originaltitel: Det guddommelige tivoli (UA in Arhus 1983); erster deutscher Titel: Die göttliche Kirmes (Erstaufführung in deutscher Sprache in St. Gallen 1995); deutscher Titel seit 2007: Der göttliche Tivoli (Lübeck 2007 und Bern 2008). Mit Ausschnitten dieser Oper und dem frühen Chorwerk Wie ein Kind (1981) gestalteten Ivan Hansen und Per Nørgård 2007 eine dramatische Chor-Montage für einen Schauspieler und Kammerchor mit dem Titel Wölfli - et udrangeret ulykkestilfceld (Wölfli - ein ausrangierter Unglücksfall).

48 Wölflis Bilder und Texte werden von Per Nørgård auch im Chorwerk Wie ein Kind (1981) und in der vierten Sinfonie Hommage à Adolf Wölfli. »Indischer Roosengarten und Chinesischer Hexensee« (1981) verarbeitet. 
und Collagen, vor allem aber die Papierberge der circa 25 ooo beschriebenen Blätter zeigte. Als ich Aperghis gleich im Anschluss an den Besuch traf, wirkte er wie benommen von der Arbeitswut, die hinter diesem Gesamtwerk steht.

Eigentlich ist die Wölfli-Kantata die Vertonung dieses unermüdlichen Schaffens: Gerade die Chorteile sind Wut- und Protestgesänge, die wie stürmische Wortwellen das Publikum überrollen, als gälte es, die Mauern der kleinen Zelle niederzureißen, in der Wölfli dies alles geschaffen hatte. Einen Abend lang müssen der Chor und das Solisten-Ensemble rastlos Wölflis Texte singen und sprechen..$^{49}$

Aperghis nennt das Textbuch zur Komposition »texte d'après Adolf Wölfli«. Er zerlegt darin Wölflis Texte in einzelne Wörter und Silben und fügt diese frei zusammen. Ein übergeordneter Sinn ergibt sich deshalb in den ersten vier Teilen der Kantata kaum, einzig bei Die Stellung der Zahlen sind einzelne Zahlen herauszuhören.

Aperghis komponiert in der Wölfli-Kantata (vgl. Abb. 24) einen außergewöhnlichen Hoquetus für sechs Stimmen. Beim Hoquetus - der Name stammt vermutlich vom französischen Begriff $>$ hoquet $<$ (Schluckauf) - handelt es sich um eine Satztechnik der mittelalterlichen Mehrstimmigkeit, bei der sich in der Regel zwei Stimmen in hohem Tempo abwechseln. Während die eine Stimme singt oder spielt, pausiert die andere, sodass die Wirkung einer einzigen, rhythmisch raffiniert verwobenen Melodie entsteht. Aperghis überträgt dieses Verfahren auf sechs Stimmen, die eine Melodie und einen Text vortragen: Die Melodie bewegt sich in Wechselbewegungen vierteltönig im Tonraum der Quarte $d^{1}-g^{1}$. Aperghis verzichtet auf rhythmische Diskontinuitäten und lässt die Stimmen in einer regelmäßigen 32tel-Kette wie Zahnräder ineinandergreifen. Jeder der sechs Stimmen sind Tonhöhen im Abstand von Viertel- oder Halbtonsekunden zugeordnet.

$\begin{array}{ll}\text { 1 Koloratursopran: } & f^{1}-f i s^{1} \\ \text { 2 Mezzosopran: } & e^{1-}-e^{11 / 4} \\ \text { 3 Countertenor: } & g^{1-}-g^{11 / 4} \\ \text { 4 Tenor:50 } & f i s^{1}-f^{13 / 4} \\ \text { 5 Bariton: } & d^{11 / 4-d^{13 / 4}} \\ \text { 6 Bass: } & d^{1-d^{11 / 4}}\end{array}$

49 Das Werk liegt in einer hervorragenden, 2014 mit dem Preis der deutschen Schallplattenkritik ausgezeichneten Aufnahme vor. Vgl. Aperghis: Wölfli-Kantata (2014).

Das vierte System (Tenorstimme) ist eine Oktave tiefer zu lesen. 


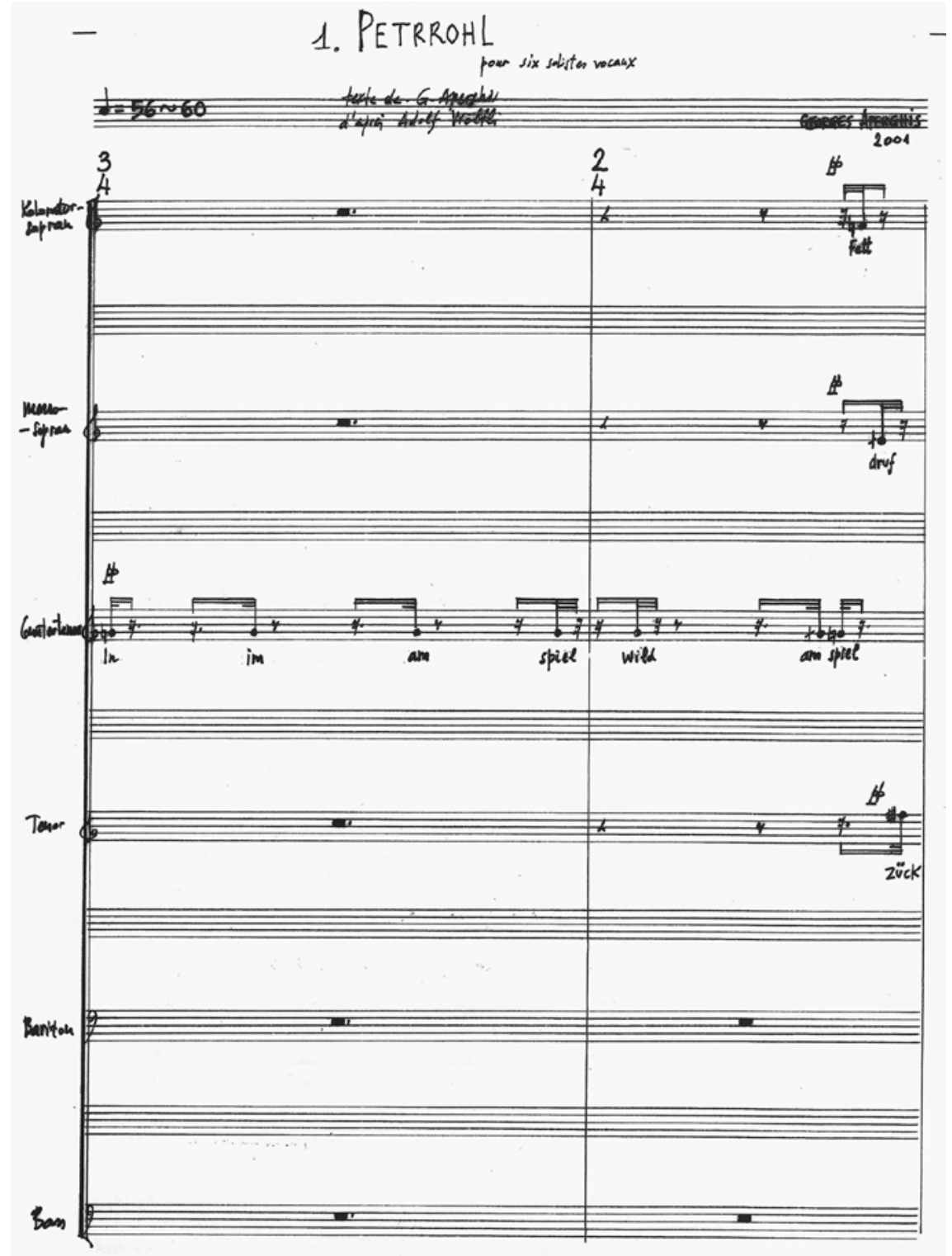

Abb. 24 Georges Aperghis: Petrrohl, S. 1f. 


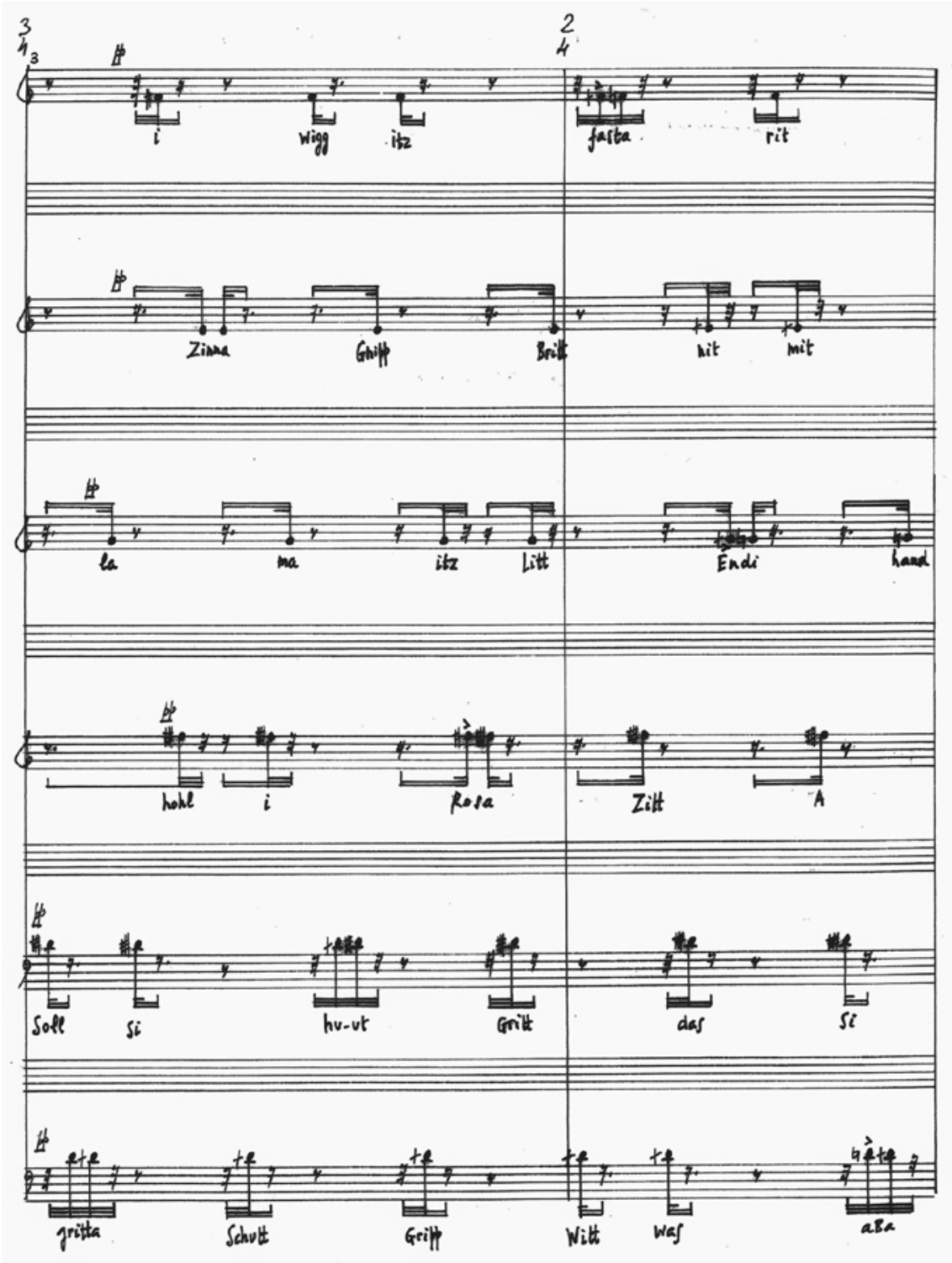


Wie Heinz Holliger zu Beginn der Oper Schneewittchen fünf verschiedene Stimmregister auf dem Ton $d^{1}$ vereinigt, zwingt auch Aperghis die sechs Stimmen, die einen Gesamtumfang von vier Oktaven abdecken könnten, in den Klangraum einer um einen Viertelton erweiterten Quarte. Er erreicht damit einen ähnlichen Mischungseffekt wie Holliger, weil die tiefste Stimme (6) im oberen und die höchste Stimme (1) im unteren Bereich ihres Umfangs singen.

Die Verteilung und Abfolge der Wörter in diesem Tonraster ist im Ausschnitt von Abbildung 24 streng geregelt. Aperghis verwendet folgende Formel: Nach dem kurzen Solo des Countertenors werden siebenmal sechs Töne von fünf der sechs Stimmen gesungen, bis das Modell wieder von vorne beginnt. Am Schluss einer >Periode`steht immer ein zweisilbiges Wort, das zwei Töne beansprucht. Jene Stimme, die in einer Zeile nicht singt, eröffnet jeweils die folgende. Am Ende schließt sich der Permutationskreis, weil die erste und die letzte $>$ Periode $<$ in Bezug auf die Verteilung der Stimmen (nicht aber vom Text her) identisch sind.

$\begin{array}{lllllll}\text { Stimmen: } & \mathbf{1} & 2 & 4 & 5 & 6 & \\ \text { Text: } & \text { fett } & \text { druf } & \text { zück } & \text { soll } & \text { gritta } & \text { (fehlende Stimme: 3) } \\ & \mathbf{3} & 5 & \mathbf{1} & 4 & \mathbf{2} & \\ & \text { la } & \text { si } & \text { i } & \text { hohl } & \text { zinna } & \text { (fehlende Stimme: 6) } \\ & \mathbf{6} & 4 & 3 & \mathbf{1} & 5 & \\ & \text { schutt } & \text { i } & \text { ma } & \text { wigg } & \text { hu-ut } & \text { (fehlende Stimme: 2) } \\ & \mathbf{2} & \mathbf{1} & 6 & 3 & 4 & \\ \text { Gnipp } & \text { itz } & \text { Gritt } & \text { itz } & \text { Rosa } & \text { (fehlende Stimme: 5) } \\ & \mathbf{5} & 3 & 2 & 6 & 1 & \\ \text { Gritt } & \text { litt } & \text { Britt } & \text { Witt } & \text { fasta } & \text { (fehlende Stimme: 4) } \\ & \mathbf{4} & 6 & 5 & 2 & 3 & \\ \text { Zitt } & \text { was } & \text { das } & \text { hit } & \text { Endi } & \text { (fehlende Stimme: 1) } \\ & \mathbf{1} & 2 & 4 & 5 & 6 & \\ \text { Rit } & \text { mit } & \text { A } & \text { si } & \text { aBa } & \text { (fehlende Stimme: 3) }\end{array}$

Diese strenge Struktur bringt in die regelmäßige 32tel-Reihung eine nervöse Unrast, die durch das geradezu rasende Tempo - MM Viertel 56-6o, also circa eine Achtelsekunde pro 32tel-Note - noch verstärkt wird. Bei gewissen Silbenfolgen vermeint man beim Lesen der Partitur deformierten semantischen Sinn zu erkennen, etwa bei »Gnipp itz Gritt itz Rosa«. Solcher Sinn ist von Aperghis nicht beabsichtigt und entsteht zufällig durch das permutierte Sprachmaterial von Wölfli. Wegen des schnellen Tempos ist ein wörtlicher Nachvollzug ausgeschlossen, wir nehmen nur ein Flimmern von Phonemen wahr. Damit trifft Aperghis neben der Energie auch den Grundklang von Wölflis Sprache, die stark mit den Vokalformanten arbeitet, dem Phänomen also, dass die Vokale 


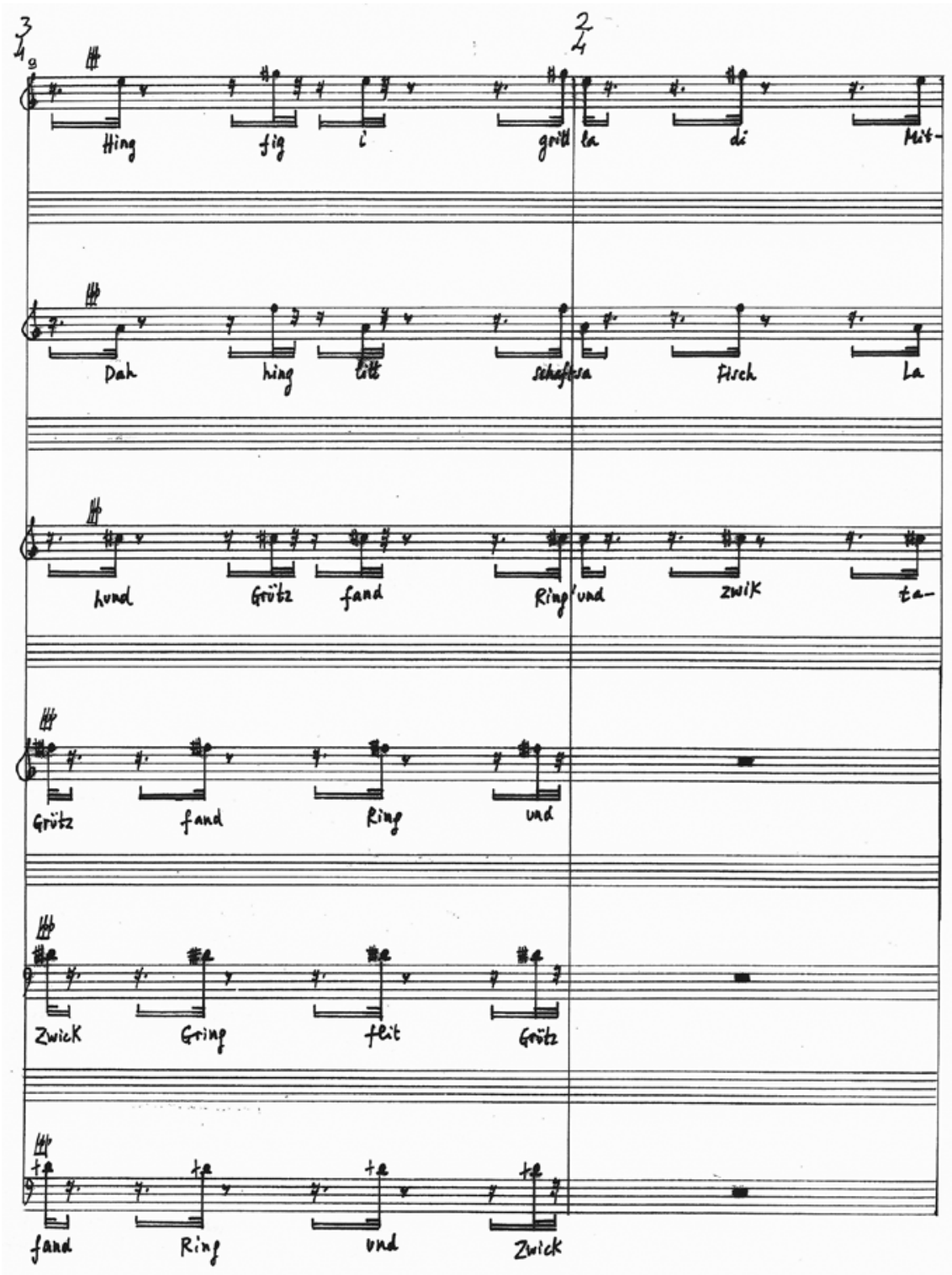

Abb. 25 Georges Aperghis: Petrrohl, S. 5

unterschiedliche Klangspektren und unterschiedliche >Tonhöhen< aufweisen. So kann im tiefen Bassbereich ein i bloß erahnt werden, ebenso ein $\mathrm{u}$ im hohen Diskant. Bei Wölfli dominieren neben plosiven Konsonanten i- und ü-Vokale, also der oberste Vokalformant, a-Vokale wirken bei Wölfli wie Periodisierungen in dieser i- und ü-Dominanz. Auch im obigen Beispiel besteht die Hälfte der 
Silben (21 von 42) aus i- oder ü-Vokalen; 12 Silben beruhen auf a-Vokalen, die verbleibenden neun Silben verteilen sich auf die Vokale u, o und e. ${ }^{51}$

Wenig später bildet Aperghis auch phonetische >Akkorde $<$, die ebenfalls permutiert werden (vgl. Abb. 25). In den drei tiefen Stimmen ist der vierte >Akkord< teilweise eine phonetische Umdrehung des ersten, und zwischen Bass- und Tenorstimme gibt es einen enggeführten phonetischen Kanon. Was die Tonhöhen betrifft, singen die tiefen Stimmen immer noch dieselben Töne wie zu Beginn (vgl. Abb. 24), während die drei hohen Stimmen abwechselnd einen A-Dur- und einen Cis-Dur-Akkord intonieren. Dass es sich dabei keineswegs um tonale Musik handelt, zeigt bereits das enharmonisch verwechselte $f^{2}$ statt eis ${ }^{2}$ beim Mezzosopran.

Auch diese Stelle ist in Hoquetus-Technik komponiert, hier aber >zweistimmig $<$, weil die Akkorde der beiden Stimmgruppen gegeneinandergesetzt sind. Im folgenden Schema ist die rhythmische Struktur auf der Basis von $3^{2}$ teln dargestellt, gelb sind die Pausen markiert, grün bzw. blau die Einsätze.

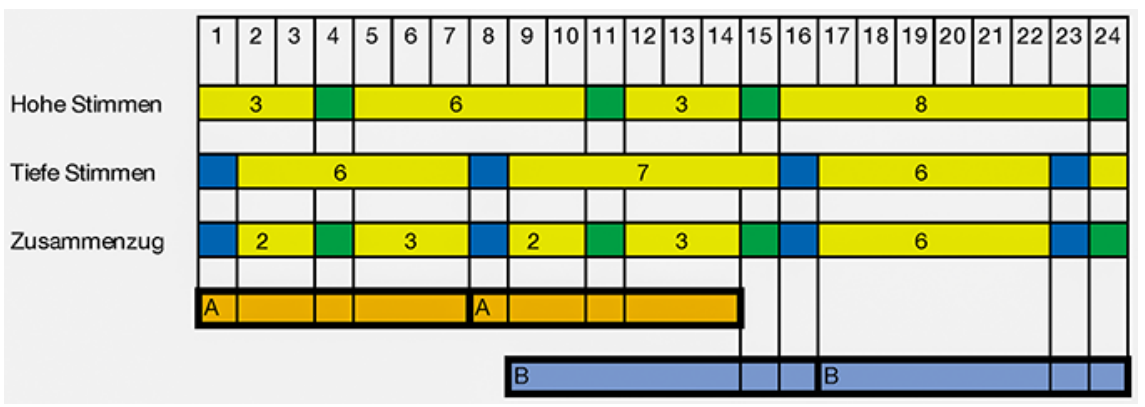

Abb. 26 Rhythmische Struktur der Stimmgruppen in Georges Aperghis' Petrrohl

Die beiden Stimmgruppen weisen eine je eigene Periodizität auf: Die Oberstimmen haben zwischen den Einsätzen jeweils eine kurze und eine lange Pause. Bei den Unterstimmen sind die Pausen annähernd gleich. Wenn man die Einsätze der beiden Stimmgruppen addiert, entsteht eine neue, gegensätzliche Periodizität, zuerst zwei Perioden A in gleichmäßiger Bewegung, dann zwei Perioden B, an deren Ende zwei Akkorde sich im 32tel-Abstand folgen, was dem Hoquetus einen veritablen `Schluckauf<-Effekt verleiht.

51 Interessanterweise gibt es diese Kompositionsweise mit Vokalformanten auch in jener Epoche, in der der von Aperghis oft verwendete Hoquetus entstand: in der Pariser NotreDame-Schule (frühes 13. Jahrhundert). Da wurde teilweise minutenlang auf einem einzigen Vokal gesungen und jeder Vokalwechsel wirkte wie eine Uminstrumentierung. 
Der Anfang der Wölfli-Kantata zeigt die Einfachheit und Klarheit, mit der Aperghis komponiert. Man kann innerhalb des rhythmischen Skandierens präzise jeden Klang wahrnehmen. Alle Elemente werden verständlich und nachvollziehbar exponiert und einander überlagert. Es ist gerade diese Fasslichkeit, die das Räderwerk dieser Musik so komplex macht. Deshalb sind die Partituren von Aperghis auch so schwierig und herausfordernd für Interpretinnen und Interpreten. Es gibt da kein Ungefähr und keine Approximationen wie bei zahlreicher überkomplexer Neuer Musik; man hört es schlicht als Fehler, wenn etwas nicht präzise realisiert ist.

Wenn Aperghis dieses Kompositionsprinzip auf die 36 Stimmen des SWRVokalensembles überträgt, erinnert die Grafik der Partitur zuweilen an die vollgeschriebenen Bildwelten des Malers Adolf Wölfli (vgl. Abb. 27). Solch eine Partitur wird auch zu einer Form von >totaler Institution<, der sich alle Mitwirkenden bei der Einstudierung unterwerfen müssen, ohne das Ganze mitprägen zu können. Alle Stimmen bleiben parzelliert; das für Chormusik so typische >Wir«-Gefühl, bei dem alle Stimmen zu einem Kollektiv verschmelzen, bleibt weitgehend aus.

\subsection{Der Ausbruch aus der totalen Institution}

In den meisten Werken von Aperghis gibt es das Phänomen des Ausbruchs, das nach Goffman für die totale Institution konstitutiv ist, weil der Ausbruch die Totalität der Institution erst zeigt. Bei solchen Ausbrüchen werden die von Aperghis installierten Kompositionsprinzipien für einen Moment verlassen. Es sind `Spielaustritte<, die eine Reflexion und damit auch eine Neuinterpretation der gesamten Komposition erlauben. Bei der Wölfli-Kantata ist der letzte Teil ein solcher Ausbruch. Chor und Solistenensemble kommen hier zwar zusammen, aber es werden keine gewaltigen Klangmassen entfaltet, vielmehr handelt es sich im Wesentlichen um ein Solo des Countertenors, das von den anderen Solistinnen und Solisten wie in einem ariosen Accompagnato bei Johann Sebastian Bach begleitet wird. Der gesungene Text ist hier klar als solcher zu erkennen und wird quasi als Monodie und Hoquetus-ähnliche Zergliederung vorgetragen. Die wenigen Choreinwürfe evozieren die Turba-Chöre barocker Passionen und Oratorien, mit denen an der Handlung beteiligte Menschengruppen repräsentiert werden. Damit eignet dem Schluss etwas Theatralisches - er könnte ein musikalischer Kommentar sein zu einer Fotografie aus dem Jahre 1921, auf der ein Krippenspiel in der Klinik Waldau abgebildet ist, bei dem Wölfli schwarz geschminkt 
$-14-$

40

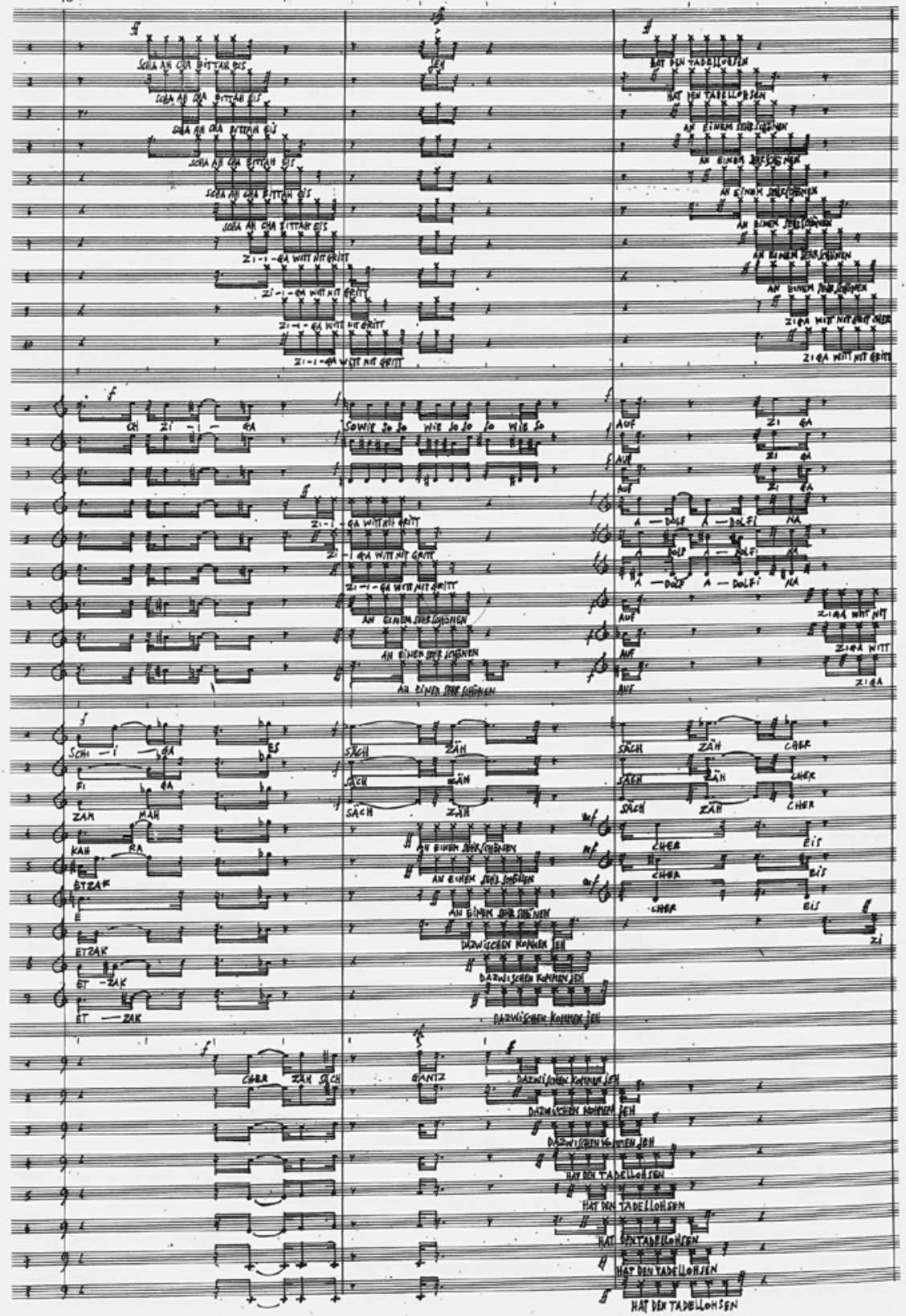

Abb. 27 Georges Aperghis: Trauer-Marsch, S. 14f. 
431

$-15$

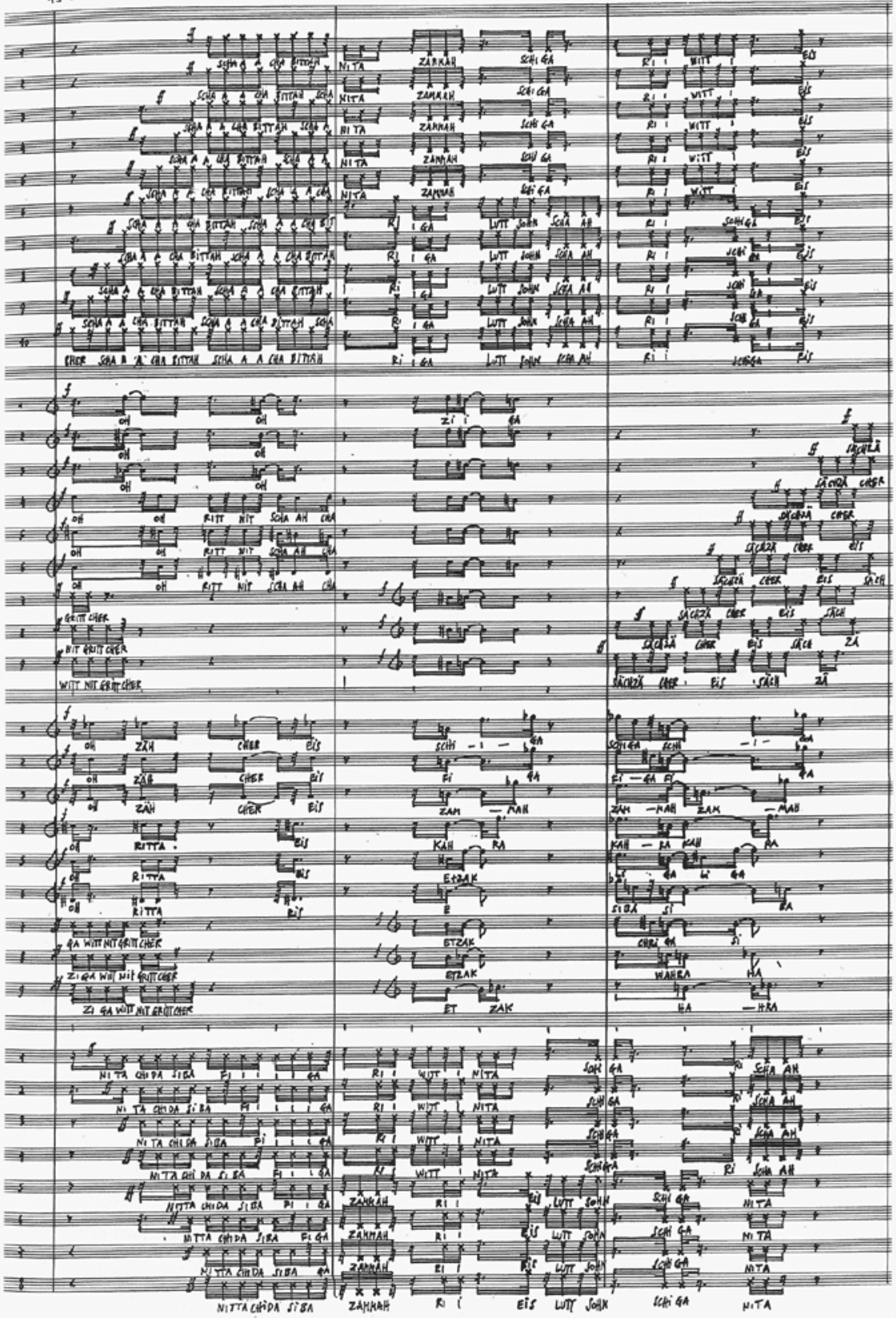


den >Mohrenkönig〈 spielt. ${ }^{52}$ Regie bei diesem Weihnachtsspiel ${ }^{53}$ führte einer der größten Theaterreformer der damaligen Zeit, Adolphe Appia, der sich 1921 und 1922 zweimal in der Waldau wegen seiner Alkoholsucht behandeln ließ. ${ }^{54}$

Das in Prosa niedergeschriebene Gedicht ließ sich Aperghis ins Französische übersetzen; es wirkt wie die Deformation eines barocken Weihnachtslieds. Es stammt aus dem >Motto<, also dem Anfang von Wölflis fiktiver Autobiografie:

Was wollen Sie, mit meinem Kind: Es ist ja alle, Tage Blind: Hat weder Haus, noch Heim: Es geht zu einem, argen Find: Der zwikt Es einmal, fest zum Grind: Diess ist der schönste, Reim: Oh frischer Muht, oh junges Bluht: Komm Heim mein Kind, komm Heim. ${ }^{55}$

Wenn man den Text in die durch die Reime suggerierte Gedichtform bringt, ergibt sich eine strenge Struktur:

Was wollen Sie, mit meinem Kind:

Es ist ja alle, Tage Blind:

Hat weder Haus, noch Heim:

Es geht zu einem, argen Find:

Der zwikt Es einmal, fest zum Grind:

Diess ist der schönste, Reim:

Oh frischer Muht, oh junges Bluht:

Komm Heim mein Kind, komm Heim.
8 Silben

8 Silben

6 Silben

8 Silben

8 Silben

6 Silben

4 Silben

4 Silben

6 Silben

52 Baumann: Adolf Wölfli im Lauf der Zeit, S. 213 und 215.

53 Zur Bedeutung des Theaterspiels und der Weihnachtsfeiern in psychiatrischen Kliniken vgl. Goffman: Asyle, S. 99-102.

54 Bablet-Hahn: Jalons biographiques, S. 9-11. Leider sind in den Nachlässen von Adolf Wölfli (Kunstmuseum Bern) und Adolphe Appia (Schweizer Archiv der darstellenden Künste SAPA, Bern) keine Dokumente zu diesem Theaterereignis erhalten. Appia erwähnt in den erhaltenen Briefen aus der Waldau das Anstaltsleben nicht. Gegenüber dem Regisseur Oskar Wälterlin in Basel schreibt er »je suis à la retraite pour ma santé.« (Appia: Postkarte vom 17.11.1921), und in einem Brief aus der Waldau an den von ihm verehrten Houston Stewart Chamberlain in Bayreuth, Theoretiker der arischen Rasse und Schwiegersohn Richard Wagners, steht: »Pardonnez à mon long silence. Je n'ai pas été très bien, et de nouveaux malaises m'ont décidé à venir ici, dans une maison dont on m'avait souvent dit grand bien. (Der Brief ist nicht datiert, muss aber im Spätherbst geschrieben worden sein; Appia: Undatierter Brief). Die inszenatorische Mitarbeit von Appia an dieser Aufführung wurde von Daniel Baumann erstmals ausgeführt. Er bezieht sich dabei auf Informationen des Schriftstellers Michel Beretti und des Künstlers Armin Heusser. Vgl. Baumann: Adolf Wölfli im Lauf der Zeit, S. 213 und Anmerkung 6, S. 223.

Wölfli: Von der Wiege bis zum Graab, Bd. 1, S. 7 . 
Vier Reime auf »ind « mit achtsilbigem Vers, drei auf »eim « mit sechssilbigem Vers und zwei auf »uht« mit viersilbigem Vers. Unter Wölflis zahlreichen Gedichten, in denen das Wort Kind vorkommt, sticht das von Aperghis ausgewählte durch seine Reime hervor. Normalerweise reimt Wölfli auf >Kind $<$ mit $>$ Rind $<$, und für die Bezeichnung >Grind < (Kopf) benutzt er meist die Emmen-

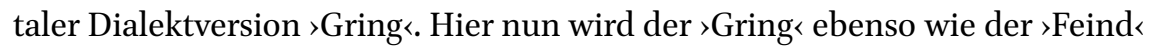
( $>$ Find $\varsigma$ ) in Wölflis perfektionistisches Reimsystem gepresst. In den Reimen des gesamten Gedichts erscheint wie in einer Art Umriss ein Wölfli-Selbstporträt: das Viereck $>$ Kind $<->$ Blind $<->$ F $[$ e $]$ ind $<->$ Grind $<$ als Konstanten in Wölflis Dasein und Schreiben, >Muht $<->$ Bluht $<$ spielt auf den ritterlichen Topos an, aber auch auf die »Unfälle«, wie er die sexuellen Übergriffe auf immer jüngere Mädchen nannte; ${ }^{56}$ und >Heim< $->$ Reim $<$ sind sein neues Zuhause: Schreiben als Zufluchtsort.

Mit diesem Schluss der Wölfli-Kantata führt Aperghis in die totale Institution zurück. Die gewaltigen Ausbrüche sind abgeklungen. In durchaus beengender Weise lässt er die inneren Zweifel und Nöte des pädophilen Patienten Wölfli aufscheinen. Um diese pädophile Veranlagung wird in vielen Wölfli-Vertonungen ein weiter Bogen gemacht. Aperghis nimmt sie ernst und gestaltet eine berührende Auseinandersetzung mit der psychischen Not eines Menschen. Die geschlechtsneutrale und alterslose Stimme des Countertenors erlaubt es ihm, in einer >Mehrfachbesetzung \ die drei KindEmanationen in Wölflis monumentaler Autobiografie Von der Wiege bis zum Graab (1908-1912) zusammenzuschweißen: zuerst das Kind Adolf (>Döufi<), dann den sich als Kind darstellenden Ich-Erzähler und schließlich das unschuldige Kind als erotisches Wunschobjekt. Das führt zu einem bedrückenden semantischen Oszillieren, dem man sich auch als Publikum schwer entziehen kann.

Im Gegensatz zum Anfang der Wölfli-Kantata mit dem zerrissen-virtuosen Hoquetus komponiert Aperghis hier schon fast ostentativ homophon. Der Chor passt sich in die rhythmische Deklamation des Solisten ein, immer zurückhaltend im Piano; das Ensemble der Solostimmen singt dazu ein Lamento, das an späte Renaissance-Polyphonie erinnert. Es ist ein komponiertes Mitleiden, bis der Countertenor das Werk in einem stockenden Solo beendet. 


\subsection{Die virtuelle Begegnung von Robert Walser und Paul Klee}

2006, ein Jahr nach Fertigstellung der Wölfli-Kantata, komponierte Georges Aperghis im Auftrag der Hochschule der Künste Bern HKB und der Wittener Tage für neue Kammermusik das Théâtre musical Zeugen für Sopran, einen Puppenspieler, fünf Instrumente und sieben Handpuppen von Paul Klee. Die Produktion wurde später auch am Warschauer Herbst und an der Biennale Venedig gezeigt. Ich war damals Leiter des Fachbereichs Musik der Hochschule der Künste Bern und für die Planung und Produktion verantwortlich. Die folgenden Ausführungen haben deshalb eine persönliche Note, die hier nicht verhüllt werden soll.

In ersten Vorgesprächen mit Georges Aperghis diskutierten wir das Projekt in zwei Richtungen: Die eine Idee war, ein Dramolett von Robert Walser zu vertonen; ich schlug Die Knaben (SW 14, 7-17) und das Dialektstück Der Teich (SW 14, 119-132) vor. Gerade dieser einzige im Schweizer Dialekt geschriebene Text von Walser, den Aperghis in der französischen Fassung kannte, ${ }^{57}$ schien mir nach dessen Faszination für Adolf Wölflis Sprachklang ein geeignetes Material zu sein. Auch war ich gespannt, wie Aperghis die geniale Mise-enabyme-Szene in Der Teich umsetzen würde, in der Fritz den gerade überstandenen Konflikt mit Messer, Gabel, Löffel und einem Tintenfleck auf dem Tischtuch als Teich nochmals nachspielt.

In eine andere Richtung wies ein Projekt mit den Handpuppen, die Paul Klee zwischen 1916 und 1923 für seinen Sohn Felix aus unterschiedlichen Materialien hergestellt hatte. Als ich Aperghis erzählte, dass Andreas Marti, der Gründungsdirektor des Zentrum Paul Klee in Bern, ein Theaterprojekt mit den Puppen unterstützen würde, löste das beim Komponisten eine ganze Reihe von Ideen aus. Er hatte die Klee-Puppen schon viele Jahre vorher im Kunstmuseum Bern als Gruppe ausgestellt gesehen; dieser Handpuppen$>$ Chor hinterließ bei ihm einen bleibenden Eindruck. Bereits 1999 hatte er sich im Werk Zwielicht mit Texten von Paul Klee auseinandergesetzt. ${ }^{58}$

57 Im Unterschied zum deutschen Sprachraum, wo Walsers Text ohne hochdeutsche Übersetzung in den gesammelten Werken vorlag, aber außerhalb der Schweiz kaum rezipiert wurde, kannte man das Dramolett im französischen Raum seit 1999 als L'Étang sehr gut. Erst 2014 wurde das Dramolett von Händl Klaus und Raphael Urweider ins Hochdeutsche übersetzt und in einer zweisprachigen Version ediert, vgl. Walser: Der Teich. Szenen.

58 Aperghis: Zwielicht auf Texte von Paul Klee, Johann Wolfgang Goethe und Franz Kafka für Sopran, Schauspieler (Bariton), Flöte, Schlagzeug, Klavier, Viola und Violoncello. UA: 23.11.2002, Opéra de Nancy et de Lorraine. Die Besetzung von Zwielicht ist jener von Zeugen eng verwandt. 
Schon bald reifte der Entschluss, die Handpuppen mit Texten von Robert Walser zu verknüpfen. Vielleicht ließe sich mit den Handpuppen ein miniaturisiertes Dramolett spielen?

Aperghis wählte nach einer langen Bedenkzeit einen radikal anderen Weg, der alle Beteiligten überraschte und viele enttäuschte: Die Handpuppen stehen nicht für Kinder und Kinderspiele, sondern werden gleichsam zu uralten Greisen, die von einer anderen Welt zeugen. Und sie werden nicht gespielt, sondern stehen wie Statuen auf der Puppenbühne. Die Texte dazu fand Aperghis in den beiden Bänden der Scènes dialoguées, mit einem Teil der ins Französische übertragenen Dramolette, die im Jahr 2000 bei Edition Zoé erschienen sind und im französischen Sprachraum zahlreiche WalserAufführungen ausgelöst haben. Von den ursprünglich diskutierten Textmöglichkeiten blieb einzig ein kurzer Ausschnitt aus Die Knaben.

Das Grundkonzept von Zeugen besteht in der Rekonstruktion einer totalen Institution: Die Puppen, der Puppenspieler und die Sängerin sind in ein Puppentheater eingesperrt. Dort werden sie von einer miniaturisierten, aber professionellen Bühnenlichtanlage mit 8o kleinen Scheinwerfern beleuchtet. Über dem Puppentheater stehen zwei vergleichsweise große Screens, auf denen in Live-Videos Bildmaterial aus dem Innern der totalen Institution vergrößert gezeigt wird. Als Publikum fühlt man sich zeitweise in eine medizinische Beobachtungsstation versetzt. Gewisse Videoeinspielungen auf den Screens wurden von Aperghis vorproduziert. Da sieht man die Puppen tanzen oder aus dem Dunkel heraus wie Geister auftauchen. Im eigentlichen Puppentheater sind die Puppen fast bewegungslos, sie werden nur hin und wieder auf Ständern minimal verschoben.

Für Zeugen wollten wir möglichst viele Fachbereiche der noch jungen und ersten Schweizer Hochschule der Künste mit Gründungsjahr 2003 einbeziehen, neben der Musik auch Theater, Gestaltung und Kunst, Konservierung und Literatur. Die Werkstätten des Fachbereichs Gestaltung und Kunst bauten das Puppentheater mit Bühnenlicht. Die originalen Puppen wurden - im doppelten Maßstab vergrößert - von der Konservierungsabteilung der HKB nachgebaut. Diese Vergrößerung erlaubte eine bessere Sichtbarkeit, denn die originalen Puppen wären zu klein gewesen, um von einem Publikum von 150 Personen noch differenziert wahrgenommen werden zu können. Man bemühte sich um Originaltreue und suchte nach den historischen Materialien und Stoffen, die Klee verwendet hatte. Allerdings gab es auch Grenzen, denn Klee benutzte beim Selbstporträt auch Tierknochen, die sich naturbedingt nicht in doppelter Größe finden ließen.

Was insbesondere Theaterschaffende bedauerten, nämlich weshalb ein virtuoser Theatermann wie Aperghis sich die Chance entgehen ließ, ein 
richtiges Puppentheater aufzuführen, erwies sich im Rückblick als die entscheidende Qualität seiner Arbeit. Eine Darstellung von szenischen Vorgängen oder gar die Erfindung solcher Vorgänge hätte die Handpuppen banalisiert und sie zu normalen Theaterpuppen gemacht. Aperghis wollte aber ihren Kunstcharakter bewahren; im Sinne von Walter Benjamin war ihm nicht der Ausstellungswert, sondern ihre auratische Kraft wichtig. Zwar wurde mit den Puppen einmal Theater gespielt, aber das ist lange vorbei. Aperghis inszeniert die Distanz, die man als Erwachsener erfährt, wenn man den Kasperlefiguren begegnet, die einen als Kind verzaubert haben. Es bleibt nur noch die Erinnerung daran: Die Figuren sind zu Zeugen der Vergänglichkeit geworden. Deshalb auch der Titel Zeugen. Oder wie Aperghis es den Figuren mit Walser in den Mund legt: »Wir sind Kunstobjekte, die für die nicht existieren, die kein Verständnis für uns aufbringen.« $\left(\mathrm{SW}_{17}, 383\right)$

\subsection{Die sieben Zeugen}

Die Handpuppen von Paul Klee galten lange Zeit als Marginalie in der KleeForschung. Dies änderte sich 2006 mit einer Publikation von Christine Hopfengart, in der die Handpuppen eingeordnet, einzeln beschrieben und kontextualisiert werden; auch die zerstörten Puppentheater und Bühnenbilder, die Klee schuf, werden dokumentiert. ${ }^{59}$ Insgesamt hat Paul Klee von 1916 bis 1925 etwa 50 solcher Handpuppen geschaffen; ein bedeutender Teil ist in der Würzburger Wohnung von Paul Klees Sohn Felix beim Bombenangriff der Alliierten am 16. März 1945 zerstört worden, andere gingen verloren oder wurden gestohlen. Entstanden sind die frühesten Puppen 1916, als der damals neunjährige Felix auf einem Jahrmarkt, der Auer Dult in München, ein Kasperltheater sah und selber ein solches bei sich zuhause haben wollte. Trotz seines Eintritts in die bayerische Armee am 11. März 1916 konnte Paul Klee seinem Sohn zum neunten Geburtstag am 30. November 1916 eine selbstgefertigte typische Kasperl-Familie schenken, mit den Figuren Kasperl, Gretl, Sepperl, Tod, Teufel, Polizist und Krokodil.

Nach Christine Hopfengart soll sich das nicht erhaltene Bühnenbild des ersten Kasperltheaters

aus Abbildungen der Publikationen des Blauen Reiters zusammengesetzt haben [...]. Das Kasperlspiel war damit für einen Augenblick ein kunstgeschichtlicher 
Ort, an dem Kinderspiel und künstlerische Avantgarde zusammentrafen und sich ergänzten. ${ }^{60}$

Denkbar wäre auch eine privatere, kunstgeschichtlich nicht weniger brisante Interpretation: Am 5. März 1916 bekam die Familie Klee ein Telegramm von Maria Marc, das sie über den Tod ihres Mannes Franz Marc unterrichtete; am gleichen Tag erhielt Klee den $»$ roten Zeddel«, den Marschbefehl. ${ }^{61}$ Die Collage mit Bildmaterial des Blauen Reiters könnte auch ein Memorial für die gefallenen Freunde August Macke ( $† 26$. September 1914) und Franz Marc († 4. März 1916) sein: Der Infanterist Paul Klee hätte dann sein Kind - wie einen hoffnungsvollen Angelus novus - in den zerschnittenen Bildern jener Aufbruchsepoche spielen lassen, die wegen des Ersten Weltkriegs bereits Vergangenheit geworden war.

Es ist in diesem Zusammenhang ein schauerlicher Zufall, dass von diesen ersten Handpuppen nur gerade Herr Tod den nächsten Weltkrieg süberlebt< hat und nicht zerstört wurde.

Erst nach dem Krieg baut Klee 1919 die nächsten Handpuppen; diese werden komplexer und sprengen das Rollendispositiv des Kasperltheaters. Einzelne Puppen lassen sich auch als Kommentare und stumme Botschaften des Vaters an den Sohn interpretieren, dessen Adoleszenz sie begleiteten, auch wenn gewisse Figuren wohl nicht unbedingt altersgerecht waren:

Nicht nur im Namen, auch in ihren biografischen und künstlerischen Bezügen weisen die Puppen weit über die Welt eines Kindes und Heranwachsenden hinaus. [...] Und dennoch, auch wenn Felix als Kind den Reichtum an Assoziationen, hintergründigen Bissigkeiten und biografischen Verknüpfungen nicht verstanden hat, muss er doch aus seinem ihm eigenen komischen Talent und einer ausgeprägten Spielfreude heraus Zugang zu den Figuren gefunden haben. ${ }^{62}$

Auch nach dem Wechsel von München nach Weimar ans Bauhaus führt Paul Klee den Puppenbau fort. Felix Klee tritt 1921 mit 14 Jahren als jüngster Student ins Bauhaus ein und führt dort bis zu seinem 18. Altersjahr immer wieder Puppenspiele auf, in denen auch das Bauhausleben ironisch reflektiert wird. In Weimar entstehen zwei Handpuppen, die sich auf konkrete Personen beziehen, nämlich auf die Kunsthändlerin Emmy >Galka<Scheyer und auf Paul Klee selbst. Die Selbstporträt genannte Handpuppe gehört zu den wenigen Selbstporträts, die von Paul Klee existieren; und man kann nur darüber

\footnotetext{
6o Hopfengart: Zwittergeschöpfe, S. 15 .

61 Jung: Paul Klee in Landshut 1916, S. 4.

62 Hopfengart: Zwittergeschöpfe, S. 8.
} 
rätseln, was den Vater und Meister des Bauhauses 1922 bewogen haben mag, dem damals 15 Jahre alten Sohn und Studenten im Bauhaus sein Konterfei zu schenken, damit dieser den Vater ins Kasperletheater einbaute. Für Felix Klee muss es keine Belastung gewesen sein - vielleicht ging die Idee sogar auf ihn zurück -, denn er soll den Vater mit seinem berndeutschen Schweizer Akzent mehrfach veräppelt haben. Und noch im Alter spielte Felix Klee mit den Puppen die Szene nach, in der Paul Klee von der Kunsthändlerin >Galka< (Emmy Scheyer) gedrängt wird, ein Bild des von ihr verehrten Alexej von Jawlensky zu kaufen, was dieser so lange verweigert, bis >Galka< ihm das Bild über dem Kopf zerschlägt. ${ }^{63}$

Aperghis wählte aus den 29 erhaltenen Handpuppen sieben aus. Die Zahl Sieben hat durchaus magischen Charakter: Sieben Töne des diatonischen Tonsystems, die Addition von Drei (Göttlichkeit) und Vier (Weltlichkeit), die Bedeutung der Zahl Sieben in Sagen und Märchen etc. Die sieben Figuren decken alle drei Schaffensphasen von Klees Puppenbau ab: 1916 München, 1919/20 München und 1921-1925 Weimar. Die Zahlen in eckigen Klammern beziehen sich auf die Nummerierung im Katalog der Handpuppen von Christine Hopfengart; auch alle Material- und Maßangaben sind diesem Katalog entnommen: ${ }^{4}$

[1] Herr Tod, 1916

Kopf: Gips, Leder, bemalt

Gewand: Stoff, Metallring

Höhe: $35 \mathrm{~cm}$

[9] Alte Jungfer, 1919

Kopf: Gips, bemalt

Gewand: verschiedene Stoffe (Baumwolle, Seide, Mischgewebe etc.), Metallring Höhe: $38 \mathrm{~cm}$

[11] Gekrönter Dichter, 1919

Kopf: Gips, bemalt (in den 1970er-Jahren retuschiert); Metallschmuck, Gazegewebe, rotes Webband

Gewand: zwei verschiedene Stoffe (rotes Batisttuch, teilweise bedruckt; gelber Stoff), Metallring

Höhe: $35 \mathrm{~cm}$

[18] Frau Tod, 1921

Kopf: Gips mit kräftigem Gewebeabdruck, bemalt

Gewand: Stoff (Kunstseide, in den 1970er-Jahren erneuert) mit Stoffapplikationen, Metallring

Höhe: $36 \mathrm{~cm}$

63 Vgl. Klee: Felix Klee und das Puppentheater, S. 41.

64 Vgl. Hopfengart: Paul Klee - Handpuppen, S. 111-142. 
[23] Selbstporträt, 1922

Kopf: Rindsknochen und Gips, bemalt

Gewand: Wollstoff (Umhang); zwei weitere verschiedene Stoffe (Flicken und Halstuch), Knopf, Metallring

Höhe: $38 \mathrm{~cm}$

[24] Emmy>Galka<Scheyer, 1922

Kopf: Gips, bemalt, Nussschalen, Metallperle, Kugelknopf, Fell

Gewand: Samt, Metallring

Höhe: $31 \mathrm{~cm}$

[27] Vogelscheuchengespenst, 1923

Kopf: Gips und Holz, bemalt, Holzscheiben, Wolle

Gewand: zwei verschiedene Stoffe, auf der Rückenpartie schwarzes Klebeband (neu), Metallring

Höhe: $45 \mathrm{~cm}$

Die sieben Zeugen schaffen in Zusammenhang mit Robert Walser ein komplexes Beziehungsgeflecht: Die drei männlichen Figuren thematisieren sich selbst, ihr Schaffen und das Stück, denn sowohl $>$ Paul Klee $<$ als auch $>$ Robert Walser< sind mit den Figuren Selbstporträt und Gekrönter Dichter als Zeugen präsent und Herr Tod beschäftigte in ihrem Schaffen beide gleichermaßen. Mit der Figur Gekrönter Dichter wollte Klee ursprünglich wohl den Personenkult um Stefan George veräppeln. Aperghis hingegen identifiziert diese Figur am Schluss von Zeugen deutlich mit Peter aus Die Knaben. Doppelbödig wird mit dieser einen Lorbeerkranz tragenden Puppe daran erinnert, dass im Abseits stehende Figuren wie Peter durchaus auch ihre Eitelkeit haben.

Ebenso entsprechen Klees weibliche Figuren Walsers Theaterkabinett, wobei hier mit Alte Jungfer und Vogelscheuchengespenst die schrulligen Damen überwiegen. Perfekt zu Walser passt die mondäne, besonders >hässlich< gestaltete und die Karikatur streifende Emmy >Galka<Scheyer. Sie wird während des Stücks von der Minikamera des Puppenspielers besonders genau abgetastet. Zu einer neuen Dimension in Zeugen verhilft Frau Tod, zumal Aperghis sie zusammen mit ihrem Mann als Ehepaar zeigt. Ein bürgerliches Ehepaar als >Herr und Frau Todく übersteigt sogar die Fantasie eines Robert Walsers.

Der erste Satz des Puppenspielers ist jener des rätselhaften Gastes aus dem szenischen Text Table d'hôte: »Ich sitze da, als wäre ich nicht vorhanden. Vielleicht besteht meine Rätselhaftigkeit darin, daß ich anspruchslos bin.« (SW 17, 413f.) Dieser Satz lässt sich zwar auf die bewegungslos ins Publikum 
starrenden Puppen beziehen, aber er definiert selbstreflexiv auch die Rolle des Puppenspielers, der wie ein stiller Zeremonienmeister im kleinen Puppentheater die Handpuppen nie sspielt<, sondern nur auf den Ständern langsam verschiebt. Vieles erinnert an das japanische Figurentheater Bunraku, bei dem die Spieler der Marionetten immer sichtbar sind. Man kann zusehen, wie sie bewegt werden, und doch wird man von den Figuren geradezu magisch angezogen. Eine ähnliche Magie entfalten die Klee-Puppen im Theater von Aperghis: Ihre Statik gibt ihnen eine Kraft im Ausdruck, wie sie große Pantomimen erreichen können, die während Minuten bewegungslos ins Publikum starren und gerade damit Spannung erzeugen.

Der verlangsamten und teilweise zum Stillstand gekommenen Welt der Puppen steht in Zeugen wie ein großformaler Chiasmus die meist schnelle und nervöse Welt der Sängerin und des Instrumentalensembles gegenüber.

Die Sängerin, von der man meist nur den Kopf sieht, agiert fahrig und überspannt zwischen den Handpuppen. Sie wirkt wie ein Geist, wie eine verlorene Seele, die zwischen den Puppen herumirrt. Manchmal wird sie von den Puppen beruhigt, manchmal tritt sie herrschsüchtig auf und führt Befehlsgesten aus, die aber ins Leere führen, weil die Puppen unbeweglich bleiben.

Mit einem speziellen, auf einer Gleitschiene montierten Stuhl kann die Sängerin lautlos und schnell quer durchs Puppentheater sausen, hinter den Puppen und vor dem Puppenspieler. Die Partie der Sängerin ist hochvirtuos; sie verbindet alle Zwischenstufen von Koloraturgesang bis zu einfachem Sprechen. In der Exaltiertheit, die Aperghis von der Rolle verlangt, erinnert sie auch an die hohen Soprane, deren vogelähnliches Zwitschern Walser in seinen Texten gerne bespöttelt. ${ }^{65}$ Sie spielt viele Rollen: Sie ist die Bühnenpartnerin des Puppenspielers, sie singt und spricht ganze Dialoge, sie ist Stimme und Spielpartnerin der Puppen. Sie ist aber oft auch das sechste Instrument des Instrumentalensembles.

Das trifft besonders auf die zahlreichen lautpoetischen Partien zu, die Aperghis in Zeugen äußerst vielfältig einsetzt, so als hätte er die Wortklangkaskaden der Wölfli-Kantata bruchlos weitergeführt. Aperghis erfindet in den kontrastreichen Klangwelten eine Art emotionale Zweitsprache, zum Beispiel:

he os la hou li vü scha na har iss a ja he ${ }^{66}$

Oder so konsonantisch dominiert, dass ein Singen gar nicht mehr möglich ist:

65 Am deutlichsten wohl in Maler, Dichter und Sängerin (SW 16, gof.).

66 Aperghis: Zeugen, S. 7, T. 32. 
tptp mn ka fi tptp do tptp do tptp ka fi ${ }^{67}$

In all seinen abstrakten Sprachkompositionen spielt Aperghis mit dem Risiko, dass sich Semantisches in den Vordergrund drängt, im obigen Beispiel etwa $>$ ka fi< (Schweizerdeutsch für >Kaffee $<$ ). Solche verständlichen Wörter wirken innerhalb von lautpoetischen Texturen auf unsere Aufmerksamkeit wie Magnete. Wir werden von ihnen angezogen und versuchen sofort, noch mehr zu verstehen. Aperghis liebt es, uns mit Pseudoverständlichkeit zu ködern und unsere Sehnsüchte nach Sinn zu nähren, allerdings nur, um diese später umso deutlicher zu enttäuschen.

Neu im Schaffen von Aperghis ist bei Zeugen, dass diese >Köder nicht Alltagsbegriffe, sondern Wörter oder Wortfragmente aus Walsers Texten sind. Im folgenden Beispiel wird der Satzteil »Wir sind Kunstobjekte« aus dem szenischen Text Porzellan (SW 17, 383) in eine klanglich stark an Schweizer Dialekt erinnernde Sprachtextur eingefügt:

a das-was-si itz-no-wott li dis sitt sa wohl oh soll ma hoho rug-ga. Wir sind gug ga ho Kunst-o-bjek-te! Sa schuf fas ta i britt Froh salm as-ti ${ }^{68}$

Aperghis setzt die lautpoetischen Verfahren auch ein, um den Einsatz eines Walser-Textes rhetorisch wirkungsvoll vorzubereiten. Im nächsten Beispiel wird ein Muster von acht Silben dreimal wiederholt, dann folgt die Reduktion auf die drei Elemente »ha he hi«, und in diesem 〉Groove〈 rutscht das lautpoetische Verfahren klanglich und rhythmisch nahtlos in Walsers ebenso musikalisch konzipierten Text Table d'hôte:

tik ta ke e ja he li ba tik ta ke e ja he li ba tik ta ke e ja he li ba

hik ha he ha he hi ha Mein Mann vi-sa-vis ist we-gen mir hin; ich seh's ihm an. ${ }^{69}$

Der längste lautpoetische Abschnitt leitet zum letzten Teil des Werkes über. Es ist der komplexeste, weil verschiedene Idiome - Japanisch, Ungarisch, Französisch, Italienisch und Deutsch - anklingen. Diese Laut-Erweiterung auf andere Idiome bereitet den Absturz des Touristen in den Bergen in der Szene DerJüngling in den Alpen vor und steht für die »eindringlichmahnende Sprache [der] Felswände« (SW 17, 427). Dieser Absturz wird in einer rollenden Permutation von Walsers Szenenanweisung »Er gibt infolge seiner Begeisterung nicht acht und fällt herunter« (SW 17, 427) umgesetzt und führt direkt in die

\footnotetext{
67 Ebd., S. 34, T. 224-226.

68 Ebd., S. 69-71, T. 493-505.

69 Ebd., S. 19f., T. 98-103.
} 
Szene mit dem gestürzten Touristen; die Zeilen ergeben sich aus kurzen Atempausen der Sängerin; nur nach »é su hop« gibt es zwei Takte Pause.

kémé-ssé a je ne vois je vi vrai ja-a a-mo

Hop-la le vi vrai a-mo-re e e e e zu é su hop

a-mo-re hop la fol-ge sei-ner nicht acht und si si s no no no a-mo-re

un-ter her-un-ter fällt her-unt

un-ter her-un-ter fällt her-unt-ter und fällt her

acht und fällt her-un-ter nicht acht und fällt her-unt

o nicht acht und fällt her-un-ter ${ }^{70}$

Diese Beispiele geben einen Einblick in das mehrstufige Kompositionsverfahren von Aperghis, bei dem nicht nur eine Textauswahl getroffen wird, sondern diese Texte in die sprachkompositorischen Verfahren eingearbeitet werden.

Das Instrumentalensemble besteht aus disparaten Instrumenten, die verschiedenen Traditionen entspringen und auf ihre Art >Zeugen< darstellen: Altsaxophon, ${ }^{71}$ Bassklarinette, Klavier, Akkordeon, Cimbalom und Schlagzeug mit der japanischen Holztrommel Mokubio und Herdenglocken. Jedes dieser Instrumente hat seinen eigenen musikalischen >Soziolekt: Das Saxophon verfügt über eine lange Jazztradition. Die Bassklarinette findet ihr Hauptrepertoire in der zeitgenössischen Musik; Aperghis lässt sie trotz ihres riesigen Tonumfangs meist im höchsten Register spielen und macht sie damit zu einem Sopraninstrument, das oft als instrumentaler Partner der Sopranistin auftritt. Das Cimbalom ist untrennbar mit der ungarischen Volksmusik verbunden. Mit dem japanischen Mokubio und den Herdenglocken bildet das Schlagzeug den >internationalen< Klangkörper. Klavier und Akkordeon werden in Walsers Schriften häufig erwähnt, sind aber gegensätzlich bewertet: Beim Klavier verspottet Walser das Virtuosentum, das Aufdringliche, das Hämmernde, und wenn es von Laien gespielt wird auch die spieltechnischen Unzulänglichkeiten; das Akkordeon (vgl. auch Kap. 11.9.5) umgekehrt wird in Walsers Texten viel positiver bewertet:

Von ihm umgangssprachlich und zugleich poetisch überhöhend als $>$ Handharfe bezeichnet, prägt es in seinen Werken oft die dunklen, nächtlichen Stimmungen. Akkordeon und Handorgel galten damals als >niedere< Instrumente. In Konzertsälen hatten sie nichts zu suchen, an Konservatorien wurden sie nicht

$70 \quad$ Ebd., S. 76-79, T. 541-443.

71 In der Partitur verlangt Georges Aperghis bis heute ein Tenorsaxophon. Der Saxophonist Marcus Weiss konnte ihn allerdings davon überzeugen, dass es eigentlich eine Partie für Altsaxophon sei. Deshalb wurde in allen bisherigen Aufführungen von Zeugen ein Altsaxophon verwendet. 
unterrichtet. Wegen ihres günstigen Preises und der damals noch einfachen Spieltechnik mit Standardbässen [...] wurden [sie] ein tragendes Element der Laienmusikbewegung. ${ }^{72}$

Aperghis bindet die unterschiedlichen Klangwelten von Akkordeon und Klavier eng aneinander und erzielt so verblüffende Effekte, etwa dann, wenn er die beiden Instrumente im dreifachen Pianissimo Duodezimen-Parallelen spielen lässt (vgl. Abb. 28). Die Duodezime ist mit dem Schwingungsverhältnis $3 / 1$ neben der Oktave $2 / 1$ und der Prim $1 / 1$ das konsonanteste Intervall der Musik und entsprechend verschmelzen die Instrumente in hohem Maße. ${ }^{73}$

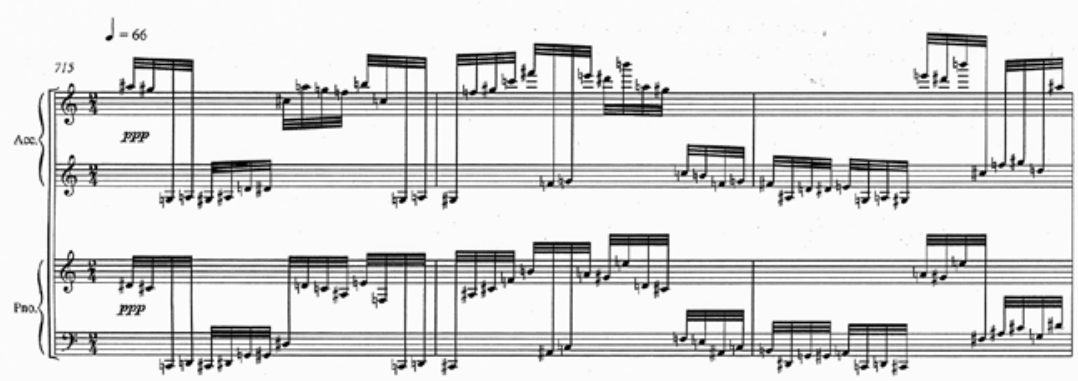

Abb. 28 Georges Aperghis: Zeugen, S. 89, T. 715-719

\subsection{Libretto und Ablauf}

Für das Libretto hat Georges Aperghis aus sechs verschiedenen szenischen Texten kurze Ausschnitte gewählt, die er meist linear aneinanderreiht. Das Werk lässt sich in drei Teile gliedern, die von einer Instrumentaleinleitung und deren Reprise am Schluss umrahmt werden. Die Teile sind allerdings kompositorisch mit Überleitungen so eng verbunden, dass die folgenden Taktzahlen nur als ungefähre Wegmarken zu verstehen sind.

\section{Einleitung}

Partitur: S. 1-10, T. 1-46

Das Stück beginnt mit einem vielgriffigen Akkordeon-Solo. Dieses Solo wird auch die Reprise zum Abschluss von Zeugen kennzeichnen. Später wird

72 Brotbeck/Sorg: Nachwort, S. 179. Vgl. auch Der Dichter (SW 4, 84f.), Der Pole (SW 4, $112-$ 114), Die Handharfe (SW 4, 139), Der Felsen (SW 4, 154-156), Der Handharfer (SW 13, 53f.).

73 Beim zweiten Viertel von Takt 715 hat der Kopist im Klavier wohl die falsche Linie erwischt, denn es müsste sicher fis statt dis stehen. 
Aperghis Klavier und Akkordeon weitgehend als monophone, also einstimmige Instrumente behandeln (vgl. Abb. 28). Die Einleitung ist eine Vorbereitung auf das japanisch geprägte Puppentheater: dicht, laut und grell. Man könnte das Ensemble von Zeugen auch als ein in die westliche Musik transferiertes Gagaku-Ensemble bezeichnen: Mit der Mundorgel Shō, dem japanischen Vorläufer des Akkordeons, der Zither Gakuso, verwandt mit dem Cimbalom, der japanischen Holztrommel Mokubio und dem Hichiriki, einem lautstarken Doppelrohrblatt-Instrument, das von Bassklarinette und Saxophon, die in höchsten Lagen spielen, imitiert wird. Auch die Sängerin singt während der Einleitung eine ans Japanische erinnernde Silbensprache.

2. Table d'Hôte (SW 17, 412-414)

Partitur: S. 10-35, T. 47-229

Lady, Lord, Straßenfegerin (Nettchen), Der Seesoldat (AdB 2, 427f.)

Partitur: S. 35-38, T. 210-243

Verarbeitung von Table d'hôte und Lady, Lord, Straßenfegerin (Nettchen), Der Seesoldat

Partitur: S. 38-46, 244-296

Aperghis verwendet zwei Textausschnitte unterschiedlicher Länge. Aus dem Text Lady, Lord, Straßenfegerin (Nettchen), Der Seesoldat, der seit dem Théâtre musical von Mischa Käser vor allem unter dem Titel Nettchen bekannt ist (vgl. Kap. 8.5.2), hat Aperghis nur eine kurze Replik ausgewählt, die es ihm erlaubt, den witzig-genialen Satz zu zitieren: »Pflichten, unaufschiebbare, rufen mich vom Milchkaffeeschauplatz ab.«(AdB 2, 428)

$\mathrm{Zu}$ Beginn dieses Teils wird die Sängerin, die während der Einleitung wie eine Instrumentalstimme wirkt, in eine Mitspielerin der Puppen gewandelt, und zwar mit einem subtilen Kniff, wie er für Aperghis typisch ist: Die Sängerin muss auf den nasalen Lauten $m$ und $n$ summen: »mnmnmn- mnmnmn mnmnmn mnmnmnmn ${ }^{7}{ }^{74}$

Während $m$ mit geschlossenem Mund artikuliert wird, muss für das $n$ der Mund zwingend geöffnet werden. Der Effekt ist auffällig: Wie ein Goldfisch im Aquarium öffnet und schließt die Sängerin während dieses Summens regelmäßig den Mund und verwandelt sich dabei in eine puppenähnliche Figur.

Der Puppenspieler sagt in Zeugen die meisten Rollen an: >Eine Frau<, >Wanderer<, >Der gestürzte Touristく etc.; das schafft Klarheit und zugleich Distanz. Seine eigenen Rollen kündet er allerdings nicht an. Er wird dadurch zum >Rätselhaften Gast $<$, dessen Rolle er in Table d'Hôte spricht. Als wortkarger Spielleiter - und auch als Vertreter der impliziten Autoren Walser und Klee 
und von dessen Handpuppen - bleibt er im Hintergrund und wirft meist nur besonders auffällige Textteile ein, zum Beispiel: »Etwas in mir klagt um das Niewiedergefundenwerdenkönnen. (SW 17, 429) Auch diese Einwürfe wirken wie Selbstreflexionen des Puppenspielers. Die Sängerin umgekehrt spricht, singt und mimt zehn verschiedene Rollen und muss mit ihrer großen Tessitura Frauen wie Männer darstellen.

Gerade wegen der zahlreichen lautpoetischen Einschübe und Verschachtelungen achtet Aperghis mit viel Respekt gegenüber den originalen Walser-Stellen darauf, dass diese deutlich deklamiert und von den Instrumenten äußerst sparsam, dafür umso präziser begleitet werden. Bereits bei der ersten gesungenen Walser-Stelle (vgl. Abb. 29) ist diese rhetorische Vertonungsweise gut festzumachen. Akkordeon und Klavier garantieren den musikalischen Fluss und klären doch den Text: Nach »Etwas« setzt das Akkordeon mit seinem Akkord einen >Doppelpunkt<; bei »lächelt und spendet« grummelt das Klavier in seiner tiefsten Lage, und auf »Beifall« erfolgt erneut der Akzent des Akkordeons und das mit Bogen gestrichene $\mathrm{C}$ des Cimbaloms. Dazu die Melodie der Sängerin mit Terzen und sogar einem Dreiklang, verbunden mit fließender Rhythmik; alles in größtmöglicher Verständlichkeit, um Walsers Text klar zu vermitteln. Die Bläser illustrieren den »Beifall« des »Etwas« (T. 55-56) erst nach diesem Text ironisch mit jubelnden Trillern, während die Sängerin schon wieder den Goldfisch mimt.

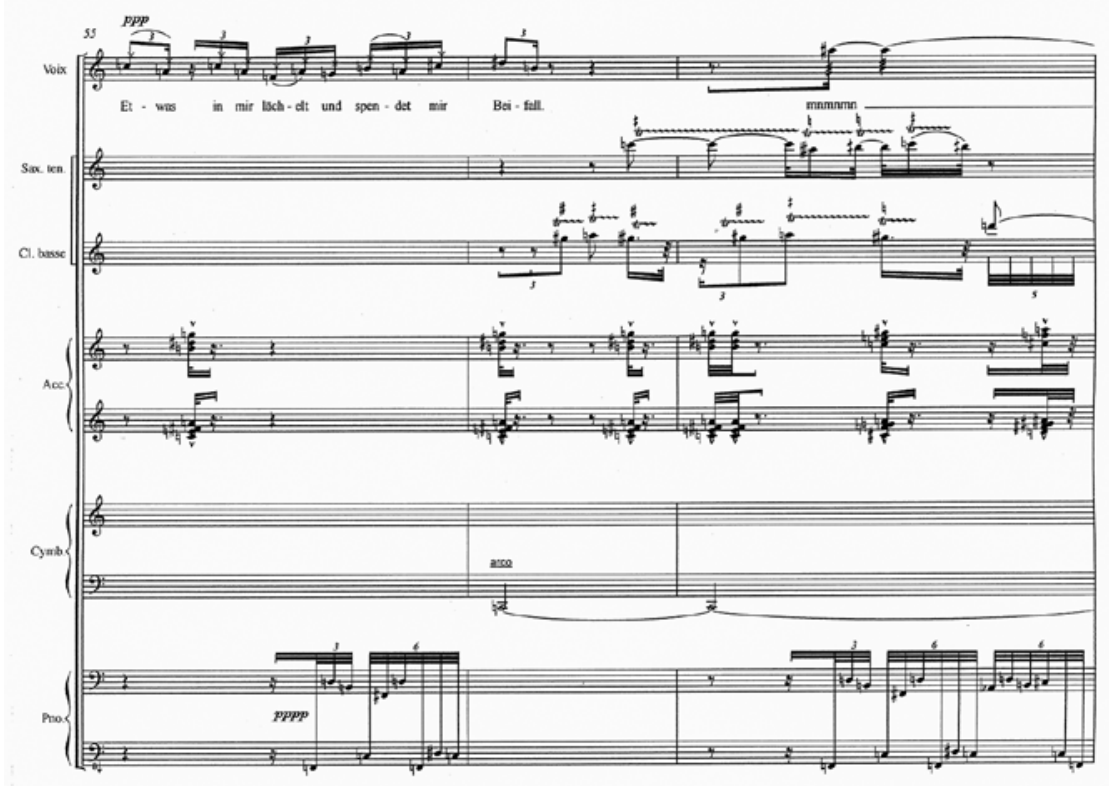

Abb. 29 Georges Aperghis: Zeugen, S. 12, T. 55-57 
Die Kreuze an den Notenhälsen in der Gesangspartie verweisen auf Sprechgesang und gehen auf Arnold Schönbergs Pierrot lunaire zurück. Während Schönberg gerade beim Pierrot lunaire immer darauf bestand, dass die Tonhöhen mit der Sprechstimme genau zu treffen seien, deutet Aperghis sowohl das Notenzeichen als auch dessen Bedeutung um. Für ihn bedeutet das Kreuz am Notenhals: »chantant en jouant (perturber le son) « ${ }^{75} \mathrm{Im}$ Vordergrund steht bei Aperghis das Theater, die Musik dient diesem zu. Damit ist nicht nur der Unterschied zu Schönberg gut beschrieben, der die Musik nie ganz dem Theater überlassen mochte, sondern auch die grundlegende Differenz vom Théâtre musical zur Oper, wo das Primat des schönen Gesangs auch nach einem halben Jahrhundert der >perturbations` durch das Regietheater noch immer gilt.

3. Nacht aus Der Wanderer (SW 17, 424)

Partitur: S. 46-6o, T. 297-410

Porzellan (SW 17, 382f.)

Partitur: S. 6o-69, T. 411-492

Der kurze Dialog von $>$ Wanderer $<$ und $>$ Passant $<$ erscheint wie die Parodie der Wanderer-Mime-Szene aus dem ersten Aufzug von Richard Wagners Siegfried, dem dritten Teil des Ring-Zyklus. Wotan besucht in der Gestalt eines Wanderers Zwerg Mime, der Siegfried erzieht. Sie stellen sich gegenseitig Fragen; Mime macht dabei den Fehler, nicht zu fragen, was er nicht weiß, sondern zu fragen, was er schon weiß. Walsers Dialog wirkt wie ein absurder Zerrspiegel dieser Szene, der Wagner-Dialog auf seine Essenz reduziert.

Wanderer: Bin ich hier auf dem rechten Weg?

Passant: Das müssen Sie selbst wissen.

Wanderer: Ich danke Ihnen für die Art, wie Sie mich auf mich selbst aufmerksam machen.

Passant: Es freut mich, daß Sie an sich glauben.

Wanderer: Sind Sie Erzieher? (SW 17, 424)

Es gibt keinen Hinweis darauf, dass Walser in seinem Leben je eine Aufführung einer Wagner-Oper besucht hat; und es ist auch nicht zu belegen, ob er das Textbuch von Siegfried kannte. Aber der Komponist Wagner taucht doch verschiedene Male in seinem Gesamtwerk auf, meist ironisch auf WagnerVerehrerinnen bezogen. ${ }^{76}$ Ebenso sind Gestalten wie Tannhäuser, Lohengrin

75 Aperghis: Zeugen, S. 1 [Notes].

76 Vgl. BA 13, 108; SW 15, 24; SW 15, 72; SW 17, 27; SW 19, 134, AdB 3, 37. 
und Siegfried in seinem Werk anzutreffen, ohne dass er sich dabei inhaltlich auf die Wagner-Figuren beziehen würde. ${ }^{77}$

In Zeugen singt die Sängerin bei diesem Dialog beide Rollen, in hoher Lage den Wanderer und in tiefer Lage mit Sprechgesang den Passanten, auch hier über einer liegenden Instrumentalbegleitung, damit der Dialog fürs Publikum klar verständlich ist.

Porzellan ist weitgehend als Melodram gestaltet; deshalb hat hier der Puppenspieler auch seine längste Sprechpartie. Im Zentrum steht der in Walsers Szene von >Dame< und >Herr gemeinsam gesprochene Satz: »Wir sind Kunstobjekte, die für die nicht existieren, die kein Verständnis für uns aufbringen.« (SW 17, 383) Für diese Szene wählt Aperghis besonders exquisite Vertonungsvarianten. Zum Beispiel komponiert er in der zweiten Hälfte von Takt 455 (vgl. Abb. 29) einen Hoquetus zwischen Klavier mit dreizehn Tönen, Sängerin mit acht Tönen und Akkordeon mit nur einem, gehaltenen Ton. Die weißen Noten in der Gesangsstimme bedeuten »chanter avec beaucoup de souffle « ${ }^{78}$ Verbunden mit den Multiphonics der Bläser in der ersten Takthälfte entsteht eine künstlich-diffizile und durchaus >porzellanene< Welt.

4. Lautpoetische Überleitung

Partitur: S. 69-81, T. 493-569

Der Jüngling in den Alpen (SW 17, 427-429)

Partitur: S. 82-97, T. 570-770

Ausschnitt aus Die Knaben (SW 14, 7-17, hier 17)

Partitur: S. 97-105, T. 770-843

Reprise

Partitur: S. 105-111, T. 844-887

Im letzten Teil erfolgt auch in Zeugen der Ausbruch aus der totalen Institution. Die Sängerin verlässt nämlich das Puppentheater und schaut gewissermaßen als >Jüngling in den Alpen< auf die Figuren im Puppentheater und auf das im Halbdunkel spielende Instrumentalensemble. Dieser Ausbruch wirkt stärker, da die Sängerin, von der bis dahin nur der Kopf im Puppentheater zu sehen war, auf einmal als ganze Gestalt sichtbar wird. Sie wirkt mächtig wie eine Riesin und überblickt nun das System, erkennt die Gesetze der totalen Institution, vermittelt implizit auch deren Kleinheit - und sstürztく dann in

77 Ich nannte mich Tannhäuser (SW 17, 45-48); Lohengrin (SW 17, 425f.); Beschaulichkeit (SW 13, 228) [Lohengrin]; Martin Weibel (SW 16, 208-213) [Siegfried, S. 211]. Gerade die letzte Frage nach dem >Erzieher erlaubt allerdings die Vermutung eines Bezugs zu Wagners berühmtem Frage-Dialog in Siegfried, denn Wotan und Mime sind beide in unterschiedlicher Weise gescheiterte Erzieher.

78 Aperghis: Zeugen, S. 1 [Notes]. 


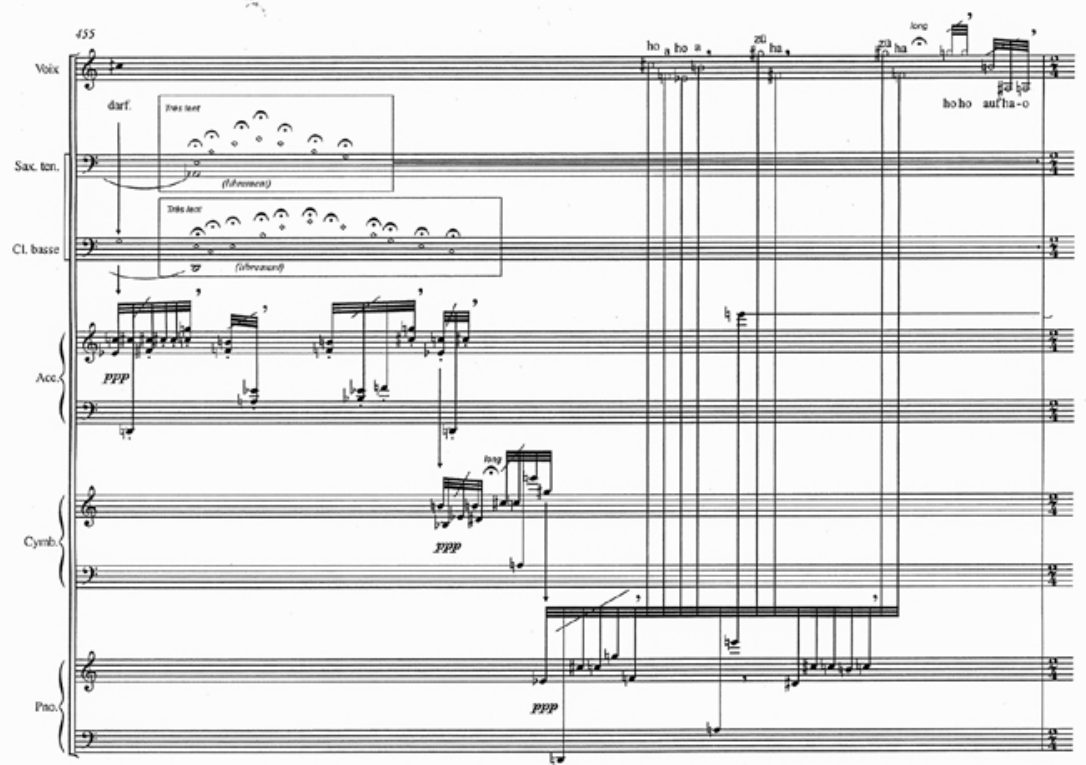

Abb. $30 \quad$ Georges Aperghis: Zeugen, S. 64, T. 455

übertragenem Sinne ab. Dieser `Absturzく wird von Aperghis in einer langen sprachmusikalischen Überleitung angebahnt. Auch das bisherige Sprachsystem bricht hier ein, denn Walsers Sprache wird mit den lautpoetischen Verfahren verschmolzen.

Der Monolog des Jünglings nach seinem >Absturz<, dargestellt in einem 'Sprachsturz<, ist als langes Vokal-Solo komponiert. In den Alpen haben die Instrumente nichts zu suchen - dieses musikalische Begleit- und Kommentarsystem wird außer Kraft gesetzt (vgl. Abb. 31). ${ }^{79}$

In diesem abschließenden Solo dominieren Vierteltöne, die auf das Unsichere und die eingeschränkte Bewegungsfreiheit nach dem Absturz hinweisen. Eine Terz aus sieben Vierteltönen bei »[im Kon]tor sein « ist das größte Intervall in dieser Sequenz. Sonst dominieren Viertelton- und Halbtonschritte. Auffällig häufig tritt daneben das erlesene Intervall von fünf Vierteltönen auf, zum Beispiel »ein Ver[unglückter]«, »uner[hörtes Glück]«, »[Wahr] heit ist«. Dieses Intervall ist ein irritierender Zwitter zwischen Sekunde und Terz, zwischen Tonschritt und Tonsprung; von im westlichen Kulturraum

79 Die Taktzählung des Kopisten ist in diesem rhythmisch frei notierten Abschnitt nicht nachvollziehbar. Am Ende des dritten Systems müsste es >unbesieglichen Unruher heißen. 


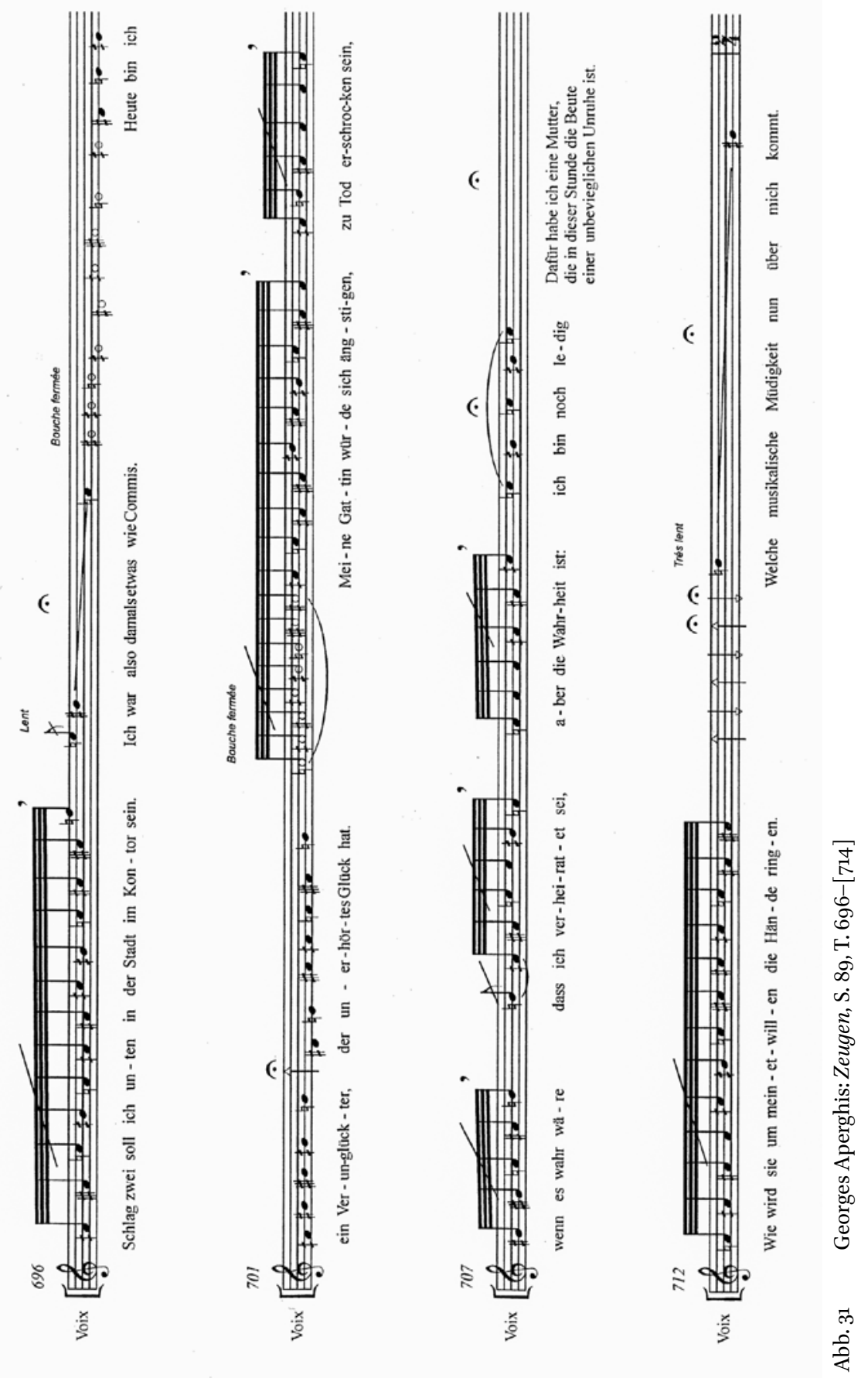


aufgewachsenen Menschen kann es nur schwer zugeordnet werden und wird deshalb als >verstimmt $<$ wahrgenommen.

Das Beispiel zeigt, wie Aperghis rhetorisch geschickt den Inhalt von Walsers Monolog im Sinne des Theaters vermittelt. Die Stelle wird mit zwei um einen Halbton verschobenen Glissandi über »Ich war also damals etwas wie Commis« und »Welche musikalische Müdigkeit nun über mich kommt« umklammert. Dort, wo ein Schauspieler Gedankenpausen einschieben würde, lässt Aperghis die Sängerin »bouche fermée« summen (vgl. Abb. 31, Mitte 1. System). Und fast schon wie ein Kasperltheater-Gag wird das »ich bin noch ledig « ausgekostet, um als weiteren Gag mit der prosaischen Sprechstimme »Dafür habe ich eine Mutter« dagegenzusetzen.

Während der darauffolgenden Vorwürfe dieser Mutter an den verunglückten Sohn heißt es demütig in die totale Institution zurückkehren. Das ist die Strafe für Ausbruch und Absturz. Für die Schlussszene greift Aperghis auf das frühe Dramolett Die Knaben zurück. Er konzentriert sich auf die Außenseiter-Figur Peter, der laut Regieanweisung »klein wie ein Hase« $(S W$ 14, 7) ist und regelmäßig von den anderen Knaben verprügelt wird. Schließlich findet Peter so etwas wie Erlösung im Tod: »Es ist jetzt Zeit zum Sterben. Mein Traum geht in Erfüllung.«(SW 14, 17)

Abrupte, zerfetzt wirkende Einwürfe der Instrumente begleiten den >Dialog<, den die Sängerin zwischen ihrem hohen, an Vogelgezwitscher erinnernden Koloraturregister und ihrer eher tiefen Sprechstimme führt. Mit einer Disco-Kugel verstreut Aperghis im Puppentheater während der Todesszene eine weihnächtliche Stimmung, die vom Akkordeon mit seinem die Reprise einleitenden Solo jäh unterbrochen wird. ${ }^{80}$ Der Anfang bildet das Ende: Aperghis wiederholt gekürzt die instrumentale Einleitung und das Stück könnte von vorne beginnen. Alles ist noch da, auch die totale Institution - und der Puppenspieler wiederholt sich:»Ich sitze da, als wäre ich nicht vorhanden. Vielleicht besteht meine Rätselhaftigkeit darin, daß ich anspruchslos bin.« (SW 17, 413f.) Dann bricht die Musik ab. Für eine totale Institution gibt es keine Schlusswendungen; es bleibt nur der Filmriss.

8o Auf der Doppel-CD des WDR 3 zu den Wittener Musiktagen 2007 ist ein längerer Ausschnitt von Zeugen festgehalten. Vgl. Aperghis: Zeugen [Auszug]. 